\title{
Thermodynamic Properties of Polybrominated/chlorinated Dibenzo-p-dioxins Calculated by Density Functional Theory
}

\author{
Xian-Wei Li, Etsuro Shibata* and Takashi Nakamura \\ Institute of Multidisciplinary Research for Advanced Materials (IMRAM), Tohoku University, Sendai 980-8577, Japan
}

\begin{abstract}
Heat capacities, standard enthalpies of formation and entropies for 339 polybrominated/chlorinated dibenzo- $p$-dioxins (PXDDs) in the gas state were calculated using the density functional theory to construct a consistent set of thermodynamic values. The heat capacities and the entropies for PXDDs increase with increase in the substitute number of bromine and chlorine. As the degree of bromination increases, the standard enthalpies of formation and the Gibbs free energies of formation of PXDDs increase, while the degree of chlorination has only a small effect on their values compared with bromination. A much higher bromine potential than chlorine potential is needed for the formation of brominated dioxin congeners. However, the bromine-chlorine equilibrium potential for the formation of PXDDs suggested thermodynamically the possibility of their priority formation under the usual gaseous conditions where $\mathrm{HBr}$ and $\mathrm{HCl}$ are similar levels, because the bromine potential of the gas is much higher than the chlorine potential when the partial pressure of the two is similar.
\end{abstract}

(Received July 1, 2003; Accepted September 3, 2003)

Keywords: polybrominated/chlorinated dibenzo-p-dioxins (PXDDs), thermodynamics, Heat capacity, entropy, enthalpy of formation, Gibbs free energy of formation, density functional theory (DFT)

\section{Introduction}

Brominated flame retardants (BFRs) including tetrabromobisphenol A (TBBPA), polybrominated diphenyl ethers (PBDEs), polybrominated biphenyls (PBBs), and hexabromocyclodecane (HBCD) have been widely used in plastics, textiles, electronic circuitry and other materials to prevent fires, and hold an important market share. Recycling activities for these consumer products containing BFRs are increasing and have become more important in recent years, due to the formation of polybrominated dibenzo- $p$-dioxins and furans (PBDD/Fs), and also mixed halogenated congeners such as polybrominated/chlorinated dibenzo- $p$-dioxins and furans (PXDD/Fs) in the case of thermal stress, because BFRs appear to be a main source of these brominated dioxin congeners in incinerators. ${ }^{1-9)}$ However, the formation behaviors of brominated dioxin congeners from the incinerators have not yet been clarified, one of the reasons is that the current analytical methods are able to detect but not quantify all mixed brominated/chlorinated congeners. Because of the complexity of analytical procedures and lack of reference standards, it has been possible to characterize and determine only a small number of PBDD/F and PXDD/F isomers.

There is much less information on PBDD/Fs and PXDD/ Fs than on their chlorinated analogues, and very little experimental data on their physicochemical properties. Using the thermodynamic data of PBDD/Fs and PXDD/Fs, it is possible to predict thermodynamically their formation in combustion gas from incineration.
We recently reported the calculated heat capacities, standard enthalpies of formation and entropies for 76 polybrominated dibenzo- $p$-dioxins (PBDDs) and 136 polybrominated dibenzofurans (PBDFs) using the density functional theory (DFT). ${ }^{10,11)}$ In this study, the thermodynamic properties were calculated for 339 PXDDs in the gas state using the DFT method with the Gaussian 98 program, ${ }^{12)}$ in order to show part of a consistent set of thermodynamic values for PXDD/Fs available for thermodynamic evaluation and consideration of their formation and decomposition in the combustion processes.

\section{Computational Methods}

Becke's three-parameter hybrid function combined with the gradient-correlation function of Lee, Yang and Parr (LYP), denoted B3LYP, was employed in the theoretical computations using DFT. The detailed method of calculating thermodynamic properties of brominated dioxins was shown in our last articles. ${ }^{10,11)}$ Heat capacity $\left(C_{\mathrm{p}}\right)$ and entropy $\left(S^{\circ}\right)$ were directly calculated using DFT and were directly obtained from the output of Gaussian programs. The equations used for calculating the absolute entropy of molecules are as follows: ${ }^{13,14)}$

$$
S=S_{\text {trans }}+S_{\text {rot }}+S_{\text {vib }}
$$

where $S_{\text {trans }}, S_{\text {rot }}$ and $S_{\text {vib }}$ are translational, rotational and vibrational entropy, respectively, which can be calculated by the following equations:

$$
\begin{aligned}
S_{\text {trans }} & =R\left[\frac{3}{2} \ln \left(\frac{2 \pi m k T}{h^{2}}\right)+\ln \frac{k T}{p}+\frac{5}{2}\right] \\
S_{\text {rot }} & =R\left[\ln \frac{\pi^{1 / 2}}{\sigma_{r}}+\frac{1}{2} \ln \frac{T^{3}}{\left(h^{2} / 8 \pi^{2} I_{x} k\right)\left(h^{2} / 8 \pi^{2} I_{y} k\right)\left(h^{2} / 8 \pi^{2} I_{z} k\right)}+\frac{3}{2}\right]
\end{aligned}
$$

*Corresponding author, E-mail: etsuro@ @agen.tohoku.ac.jp 


$$
S_{\text {vib }}=R \sum_{i=1}^{3 N-6}\left[\frac{h v_{i} / k T}{\exp \left(h v_{i} / k T\right)-1}-\ln \left\{1-\exp \left(-h v_{i} / k T\right)\right\}\right]
$$

where $R$ is the gas constant $\left(8.31451 \mathrm{~J} \cdot \mathrm{mol}^{-1} \cdot \mathrm{K}^{-1}\right), N$ is the atom number in a molecule, $m$ is the molecular mass, $k$ is the Boltzmann constant $\left(1.380658 \times 10^{-23} \mathrm{~J} \cdot \mathrm{K}^{-1}\right), h$ is Planck's constant $\left(6.6260755 \times 10^{-34} \mathrm{~J} \cdot \mathrm{s}\right), T$ is the temperature, $p$ is the pressure, $\sigma_{r}$ is the symmetry number for rotation, $I$ is the moment of inertia, and $v$ is the vibrational frequency.

The heat capacity at constant pressure was calculated using: ${ }^{13,14)}$

$$
C_{\mathrm{p}}=C_{\text {trans }}+C_{\text {rot }}+C_{\text {vib }}=\frac{5}{2} R+\frac{3}{2} R+R \sum_{i=1}^{3 N-6} \exp \left(h v_{i} / k T\right)\left[\frac{h v_{i} / k T}{\exp \left(-h v_{i} / k T\right)-1}\right]^{2}
$$

where $C_{\text {trans }}, C_{\text {rot }}$ and $C_{\text {vib }}$ are contribution to heat capacity due to translation, rotational motion, and vibrational motion, respectively.

The calculated absolute enthalpies $\left(H^{\circ}\right)$ and free energies $\left(G^{\circ}\right)$ of PXDDs, dibenzo- $p$-dioxin (DD), $\mathrm{Br}_{2}, \mathrm{Cl}_{2}$ and $\mathrm{H}_{2}$ by DFT and literature values of standard enthalpies of formation $\left(\Delta_{\mathrm{f}} H^{\circ}\right)$ and Gibbs free energies of formation $\left(\Delta_{\mathrm{f}} G^{\circ}\right)$ of $\mathrm{DD}, \mathrm{Br}_{2}, \mathrm{Cl}_{2}$ and $\mathrm{H}_{2}$ were used to obtain the $\Delta_{\mathrm{f}} H^{\circ}$ and $\Delta_{\mathrm{f}} G^{\circ}$ of PXDDs at $298 \mathrm{~K}$. Changes of enthalpy and Gibbs free energy were evaluated on the basis of the following reaction using $H^{\circ}$ and $G^{\circ}$,

$$
\begin{aligned}
& \Delta_{\mathrm{r}} H^{\circ}=\left[H^{\circ}(\mathrm{PXDD})+(\mathrm{m}+\mathrm{n}) / 2 H^{\circ}\left(\mathrm{H}_{2}\right)\right]-\left[H^{\circ}(\mathrm{DD})+\mathrm{m} / 2 H^{\circ}\left(\mathrm{Br}_{2}\right)+\mathrm{n} / 2 H^{\circ}\left(\mathrm{Cl}_{2}\right)\right] \\
& \Delta_{\mathrm{r}} G^{\circ}=\left[G^{\circ}(\mathrm{PXDD})+(\mathrm{m}+\mathrm{n}) / 2 G^{\circ}\left(\mathrm{H}_{2}\right)\right]-\left[G^{\circ}(\mathrm{DD})+\mathrm{m} / 2 G^{\circ}\left(\mathrm{Br}_{2}\right)+\mathrm{n} / 2 G^{\circ}\left(\mathrm{Cl}_{2}\right)\right]
\end{aligned}
$$

where $\Delta_{\mathrm{r}} H^{\circ}$ and $\Delta_{\mathrm{r}} G^{\circ}$ indicate the enthalpy and Gibbs free energy changes of reaction, respectively. The same values of $\Delta_{\mathrm{r}} H^{\circ}$ and $\Delta_{\mathrm{r}} G^{\circ}$ can also be evaluated using $\Delta_{\mathrm{f}} H^{\circ}$ and $\Delta_{\mathrm{f}} G^{\circ}$ of each compound in eqs. 7 and 8 . The unknown $\Delta_{\mathrm{f}} H^{\circ}$ and $\Delta_{\mathrm{f}} G^{\circ}$ of PXDD can be calculated as follows:

$$
\begin{aligned}
& \Delta_{\mathrm{f}} H^{\circ}(\mathrm{PXDD})=\Delta_{\mathrm{r}} H^{\circ}-(\mathrm{m}+\mathrm{n}) / 2 \Delta_{\mathrm{f}} H^{\circ}\left(\mathrm{H}_{2}\right)+\Delta_{\mathrm{f}} H^{\circ}(\mathrm{DD})+\mathrm{m} / 2 \Delta_{\mathrm{f}} H^{\circ}\left(\mathrm{Br}_{2}\right)+\mathrm{n} / 2 \Delta_{\mathrm{f}} H^{\circ}\left(\mathrm{Cl}_{2}\right) \\
& \Delta_{\mathrm{f}} G^{\circ}(\mathrm{PXDD})=\Delta_{\mathrm{r}} G^{\circ}-(\mathrm{m}+\mathrm{n}) / 2 \Delta_{\mathrm{f}} G^{\circ}\left(\mathrm{H}_{2}\right)+\Delta_{\mathrm{f}} G^{\circ}(\mathrm{DD})+\mathrm{m} / 2 \Delta_{\mathrm{f}} G^{\circ}\left(\mathrm{Br}_{2}\right)+\mathrm{n} / 2 \Delta_{\mathrm{f}} G^{\circ}\left(\mathrm{Cl}_{2}\right)
\end{aligned}
$$

The discrepancies between our calculated results and available experimental values for 16 brominated arenes have also been discussed and our calculated results confirmed to be accurate. ${ }^{10,11)}$ There are theoretically 1700 PXDD and 3320 PXDF isomers. As stated, 339 representative PXDDs were calculated, and the calculated isomers cover all substitution numbers of bromine and chlorine.

\section{Results and Discussion}

The calculated results of $C_{\mathrm{p}}, \Delta_{\mathrm{f}} H^{\circ}, S^{\circ}$ and Gibbs free energy of formation $\left(\Delta_{\mathrm{f}} G^{\circ}\right)$ for the 339 PXDDs in the gas phase at $298.15 \mathrm{~K}$ (Temperature range for $C_{\mathrm{p}}$ is 298.15 to $1500 \mathrm{~K}$.) and $101.325 \mathrm{kPa}$ are listed in the table in Appendix.

Figure 1 shows the average standard enthalpies and free energies of formation for each PXDD homologue within the same degrees of bromination and chlorination at $298 \mathrm{~K}$. Figure 2 shows the average entropies and heat capacities for each homologue. As the degree of bromination increases, $\Delta_{\mathrm{f}} H^{\circ}$ values of PXDDs increase, while decreasing slightly with increase in degree of chlorination compared with bromination; the values of $\Delta_{\mathrm{f}} G^{\circ}$ of PXDDs have the same tendency. It is suggested that the relative thermodynamic stability is affected by the intramolecular repulsion effect. ${ }^{15)}$ The repulsion energy of $\mathrm{Br}-\mathrm{Br}$ is much larger than that of $\mathrm{Cl}$ $\mathrm{Cl}$, which makes the molecule unstable, because the bromine atom is larger than the chlorine atom. The values of $S^{\circ}$ and $C_{\mathrm{P}}$

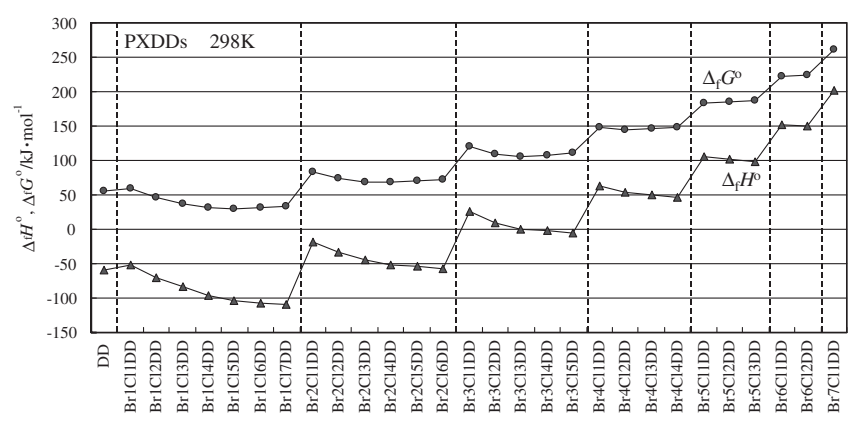

Fig. 1 Average standard enthalpies and free energies of formation for each PXDD homologue within the same degrees of bromination and chlorination at $298 \mathrm{~K}$

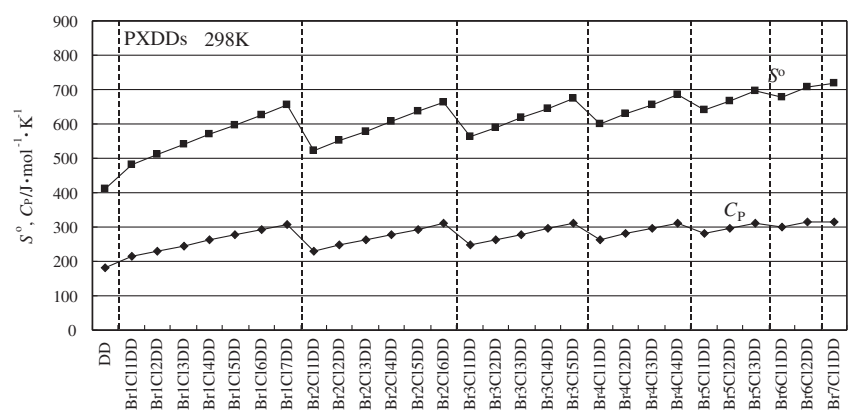

Fig. 2 Average entropies and heat capacities for each PXDD homologue within the same degrees of bromination and chlorination at $298 \mathrm{~K}$. 
Most Stable

Least Stable<smiles>Clc1ccc2c(c1)Oc1ccc(Br)cc1O2</smiles>

$2-\mathrm{Br}_{1}-7-\mathrm{Cl}_{1}-\mathrm{DD}$<smiles>Clc1ccc2c(c1)Oc1cc(Cl)cc(Br)c1O2</smiles>

$1-\mathrm{Br}_{1}-3,7-\mathrm{Cl}_{2}-\mathrm{DD}$<smiles>Clc1cc(Cl)c2c(c1)OC1C(Br)CC(Cl)CC1O2</smiles>

$1-\mathrm{Br}_{1}-3,6,8-\mathrm{Cl}_{3}-\mathrm{DD}$<smiles>ClC1=CC(Cl)C2Oc3c(Cl)cc(Cl)cc3OC2=C1Br</smiles>

$1-\mathrm{Br}_{1}-2,4,6,8-\mathrm{Cl}_{4}-\mathrm{DD}$<smiles>ClC1=CC(Cl)C2Oc3c(Cl)cc(Cl)c(Cl)c3OC2=C1Br</smiles>

$1-\mathrm{Br}_{1}-2,4,6,7,9-\mathrm{Cl}_{5}-\mathrm{DD}$<smiles>ClC1C(Br)CCC2Oc3ccccc3OC21</smiles>

$2-\mathrm{Br}_{1}-1-\mathrm{Cl}_{1}-\mathrm{DD}$<smiles>Clc1cc2c(c(Cl)c1Br)Oc1ccccc1O2</smiles>

2- $\mathrm{Br}_{1}-1,3-\mathrm{Cl}_{2}-\mathrm{DD}$<smiles>Clc1c(Cl)c(Br)c2c(c1Cl)Oc1ccccc1O2</smiles>

$1-\mathrm{Br}_{1}-2,3,4-\mathrm{Cl}_{3}-\mathrm{DD}$<smiles>Clc1c(Cl)c(Cl)c2c(c1Cl)OC1CCC=C(Br)C1O2</smiles>

$1-\mathrm{Br}_{1}-6,7,8,9-\mathrm{Cl}_{4}-\mathrm{DD}$<smiles>ClC1=CC[C@H]2OC3C(Cl)=C(Cl)C(Cl)C(Cl)C3O[C@@H]2C1Cl</smiles>

$2-\mathrm{Br}_{1}-1,3,4,6,7-\mathrm{Cl}_{5}-\mathrm{DD}$

Fig. 3 Most and least stable isomers within the monobrominated dioxin homologues on the basis of standard free energies of formation at $298 \mathrm{~K}$.

increase with increase in the substitution numbers of bromine and chlorine as shown in Fig. 2. One reason is thought to be the change in molecular weight with the substitutions of bromine and chlorine.

Figure 3 shows the most and least stable isomers on the basis of standard Gibbs free energies of formation at $298 \mathrm{~K}$ within the mono-brominated dioxin homologues calculated in this study. It is obvious that the most stable isomer has no or a minimum number of adjacent chlorine and bromine atoms, whereas the least stable isomer has a maximum number of nearest chlorines and bromines because the intramolecular repulsion energies affect the stability of the molecule.

Using the regression method, the correlations of thermodynamic properties $\left(\Delta_{\mathrm{f}} H^{\circ}, \Delta_{\mathrm{f}} G^{\circ}, S^{\circ}\right.$ and $\left.C_{\mathrm{p}}\right)$ at $298 \mathrm{~K}$ with the substitution number of bromine (x) and chlorine (y) were obtained for PXDDs from the calculated results of each isomer in the table in Appendix. The thermodynamic properties of PXDDs correlate very well with the degree of bromination (x), the obtained correlations being as follows:

For PXDDs,

$$
\begin{aligned}
\Delta_{\mathrm{f}} H^{\circ}(298 \mathrm{~K})= & (43.44 \pm 0.29) \mathrm{x}-(9.78 \pm 0.29) \mathrm{y} \\
& -(96.06 \pm 1.28), \quad \mathrm{R}^{2}=0.988 \\
\Delta_{\mathrm{f}} G^{\circ}(298 \mathrm{~K})= & (35.04 \pm 0.30) \mathrm{x}-(4.44 \pm 0.30) \mathrm{y} \\
& +(17.88 \pm 1.32), \quad \mathrm{R}^{2}=0.979 \\
S^{\circ}(298 \mathrm{~K})= & (39.10 \pm 0.07) \mathrm{x}+(28.68 \pm 0.07) \mathrm{y} \\
& +(414.62 \pm 0.30), \quad \mathrm{R}^{2}=0.999 \\
C_{\mathrm{p}}(298 \mathrm{~K})= & (16.82 \pm 0.02) \mathrm{x}+(15.77 \pm 0.02) \mathrm{y} \\
& +(181.59 \pm 0.07), \quad \mathrm{R}^{2}=0.9998
\end{aligned}
$$

where $\mathrm{R}^{2}$ indicates the coefficient of determination for the regression analysis. All these formulas indicate that the substitution number of bromine (x) has much more effect on the thermodynamic properties of PXDDs than the substitution number of chlorine (y).

Figure 4 shows the Gibbs free energy changes $\left(\Delta_{\mathrm{r}} G^{\circ}\right)$ in the brominated and chlorinated reactions of DD to $2,3,7,8$ tetrabrominated/chlorinated dibenzo- $p$-dioxins (2,3,7,8TeXDDs) shown in eq. (6) in which $\mathrm{m}+\mathrm{n}=4$. The values of $\Delta_{\mathrm{r}} G^{\circ}$ increase with increase in degree of bromination of TeXDDs. It is suggested thermodynamically that the formation of higher brominated PXDDs cannot occur as easily as the lower brominated PXDDs and PCDDs.

Figure 5 shows the bromine-chlorine equilibrium potential for the formation of 2,3,7,8-TeXDDs $\left(\mathrm{Br}_{\mathrm{m}} \mathrm{Cl}_{(4-\mathrm{m})} \mathrm{DD}\right)$ from $2,3,7,8-\mathrm{TeCDD}$ at $573 \mathrm{~K}$, where these potentials are represented by the partial pressures of $\mathrm{Br}_{2}$ and $\mathrm{Cl}_{2}$, respectively. The diagram is based on the bromination reaction of 2,3,7,8TeCDD as shown in the following:

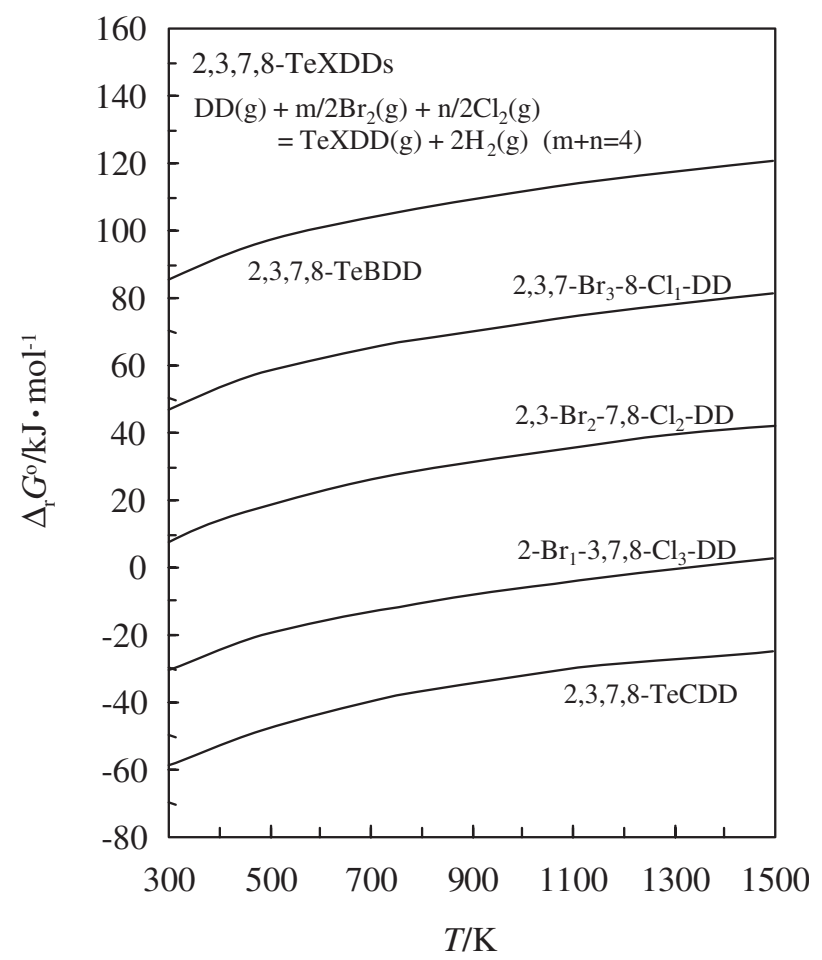

Fig. 4 Gibbs free energy changes in the brominated and chlorinated reactions of DD to $2,3,7,8-\mathrm{TeXDD}$. 


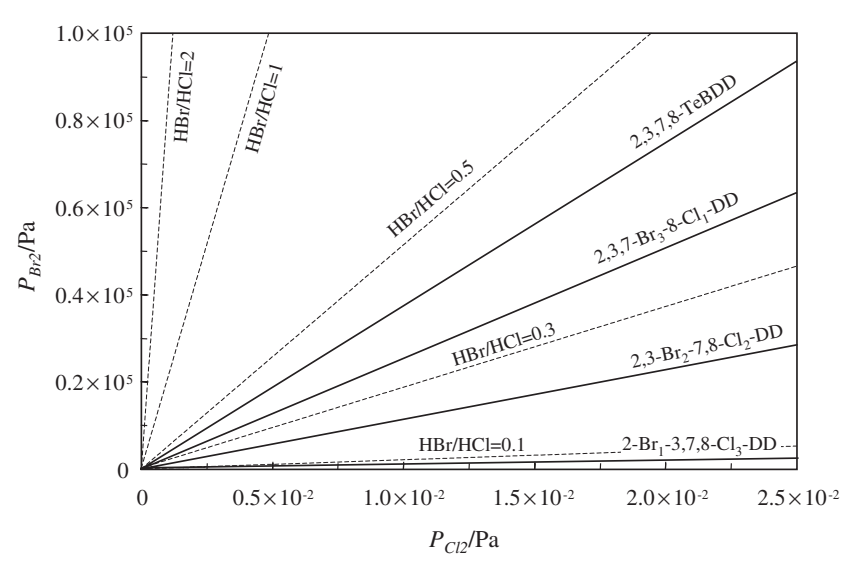

Fig. 5 Bromine-chlorine equilibrium potential for the formation of 2,3,7,8TeXDDs from 2,3,7,8-TeCDD at $573 \mathrm{~K}$.

$$
\begin{aligned}
& 2 / \mathrm{m} \mathrm{TeCDD}+\mathrm{Br}_{2}(\mathrm{~g}) \\
& \quad=2 / \mathrm{m} \mathrm{Br}_{\mathrm{m}} \mathrm{Cl}_{(4-\mathrm{m})} \mathrm{DD}+\mathrm{Cl}_{2}(\mathrm{~g}), \quad(\mathrm{m}=1,2,3,4) \\
& \Delta_{\mathrm{r}} G^{\circ}=-R T \ln K=-R T \ln \frac{P_{\left.\mathrm{Br}_{\mathrm{m} C l} \mathrm{Cl} \mathrm{m}\right)}^{2 / m} P_{\mathrm{Cl}_{2}}}{P_{\mathrm{TeCDD}}^{2 / m} P_{\mathrm{Br}_{2}}}
\end{aligned}
$$

where $K$ and $P$ indicate the equilibrium constant of reaction and partial pressure, respectively. The diagram was drawn assuming $P_{\mathrm{TeCDD}}=P_{\mathrm{Br}_{m} \mathrm{Cl}(4-m)} \mathrm{DD}$ in order to know easily the possibility of bromination of the chlorinated dioxin congeners thermodynamically. The diagram shows that each 2,3,7,8-TeXDD can be thermodynamically formed from $2,3,7,8$-TeCDD at higher bromine potential than each solid line, respectively. The equilibrium potentials of bromine and chlorine for the relationship between the gaseous $\mathrm{HCl}$ and $\mathrm{HBr}$ at their several partial pressure ratios are also shown by dotted lines based on the following reaction:

$$
\begin{aligned}
& 2 \mathrm{HCl}(\mathrm{g})+\mathrm{Br}_{2}(\mathrm{~g})=2 \mathrm{HBr}(\mathrm{g})+\mathrm{Cl}_{2}(\mathrm{~g}) \\
& \Delta_{\mathrm{r}} G^{\circ}=-R T \ln K=-R T \ln \frac{P_{\mathrm{HBr}}^{1 / 2} P_{\mathrm{Cl}_{2}}}{P_{\mathrm{HCl}}^{1 / 2} P_{\mathrm{Br}_{2}}}
\end{aligned}
$$

The priority formations for each 2,3,7,8-TeXDD and their relationship with the ratio of $\mathrm{HBr}$ to $\mathrm{HCl}$ can be distinguished and discussed using this diagram. For example, when the ratio of $\mathrm{HBr}$ to $\mathrm{HCl}$ is $0.3,2,3-\mathrm{Br}_{2}-7,8-\mathrm{Cl}_{2}$-DD is supposed to be more stable than $2,3,7,8-\mathrm{TeCDD}$ thermodynamically. From the diagram, it is suggested that much higher bromine potential than chlorine potential is needed for the formation of brominated dioxin congeners. However, even in the formation region of 2,3,7,8-TeBDD, the partial pressure of $\mathrm{HBr}$ can be lower than that of $\mathrm{HCl}$ such as $\mathrm{HBr} / \mathrm{HCl}=0.5$, because $\mathrm{HBr}$ has thermodynamically much less stability than $\mathrm{HCl}$ and the equilibrium of the reaction shown in eq. (17) shifts to the left. In other words, when the partial pressure of $\mathrm{HBr}$ is similar to that of $\mathrm{HCl}$ in the gas, the bromine potential of the gas is far higher than the chlorine potential. Although a detailed discussion on the formation of brominated dioxin congeners is difficult using Fig. 5 because of the simplified calculations, the diagram can suggest thermodynamically the possibility of their priority formation under the usual gaseous conditions where $\mathrm{HBr}$ and $\mathrm{HCl}$ are at similar levels.
Yazawa et al. ${ }^{16)}$ evaluated thermodynamically the formation and decomposition behavior of PCDD/Fs, and the effects of temperature and gas compositions on them in the $\mathrm{C}-\mathrm{H}-\mathrm{O}-\mathrm{Cl}$ quaternary combustion gas assuming homogeneous gas equilibrium. They used the consistent set of thermodynamic data of PCDD/Fs estimated by Menad. ${ }^{17,18)}$ Actually, the formation and decomposition of dioxin congeners are supposed to proceed in the heterogeneous gas solid interface, and the kinetic effects are also important and effective on their formation. The thermodynamic prediction is not necessarily agreement with the practical quantitative analysis in the incinerators. However, the thermodynamic evaluations can show us the approximate trends of the effect of gas compositions and temperature on their formation and decomposition behaviors. ${ }^{16,19)}$ Thermodynamic evaluations are useful for the formation and decomposition of dioxin congeners even for PCDD/Fs, because it is very difficult to clarify their complicated formation mechanism kinetically at present. There is much less information and fewer reports on the formation and decomposition behaviors of PBDD/Fs and PXDD/Fs in incinerators compared with PCDD/Fs, because of analytical difficulties. Using the consistent set of thermodynamic data for PBDD/Fs and PXDD/Fs, the thermodynamic evaluations and considerations would be possible for the brominated dioxin congeners in the combustion gas, and would provide important knowledge of their formation and decomposition behaviors.

\section{Conclusion}

The thermodynamic properties of 339 PXDDs in the gas state were calculated using the density functional theory (DFT) to construct a consistent set of thermodynamic values for them. As the degree of bromination increases, the standard enthalpies of formation for PXDD/Fs and the Gibbs free energies of formation of PXDDs increase, while the degree of chlorination has only a small effect on their values compared with bromination.

Much higher bromine potential than chlorine potential is needed for the formation of brominated dioxin congeners. However, the bromine-chlorine equilibrium potential for the formation of PXDDs suggested thermodynamically the possibility of their priority formation under the usual gaseous conditions where $\mathrm{HBr}$ and $\mathrm{HCl}$ are at similar levels, because the bromine potential of the gas is much higher than the chlorine potential when the partial pressure of the two is similar in the combustion gas.

\section{Acknowledgments}

This work was financially supported by a Grant-in-Aid for Waste Treatment Research from the Ministry of the Environment of Japan (No. K1431) and a Grant-in-Aid for Scientific Research (A) from the Japan Society for the Promotion of Science (No. 15206099), Japan.

\section{REFERENCES}

1) WHO: Polybrominated Dibenzo-p-dioxins and Dibenzofurans, Environmental Health Criteria 205, (World Health Organization, Geneva, 
1998) p. 205.

2) S. Sakai, J. Watanabe, Y. Honda, H. Takatsuki, I. Aoki, M. Futamatsu and K. Shiozaki: Chemosphere 42 (2001) 519-531.

3) H. Wichmann, F. T. Dettmer and M. Bahadir: Chemosphere 47 (2002) 349-355.

4) D. Sedlak, R. Dumler-Gradl, H. Thoma and O. Vierle: Chemosphere 37 (1998) 2071-2076.

5) S. K. Bindra and R. S. Narang: Chemosphere 31 (1992) 4413-4425.

6) W. Chatkittikunwong and C. S. Creaser: Chemosphere 29 (1994) 559566.

7) G. W. Sovocool, R. K. Mitchum, Y. Tondeur, W. D. Munslow, T. L. Vonnahme and J. R. Donnelly: Biomed. Mass Spectrom. 15 (1998) 669-676.

8) A. G. Schuur, F. F. Legger, M. E. van Meeteren, M. J. H. Moonen, I. van Leeuwen-Bol, Å. Bergman, T. J. Visser and A. Brouwer: Chem. Res. Toxicol. 11 (1998) 1075-1081.

9) P. M. Lemieux and J. V. Ryan: Waste Management 18 (1998) 361-370.

10) X.-W. Li, E. Shibata and T. Nakamura: J. Chem. Eng. Data 48 (2003) $727-735$.
11) X.-W. Li, E. Shibata and T. Nakamura: Mater. Trans. 44 (2003) 10041013.

12) Æ. Frisch and M. J. Frisch: Gaussian 98 User's Reference; second ed. (Gaussian, Inc., Pittsburgh, 1999) pp. 71-76.

13) D. A. McQuarrie and J. D. Simon: Molecular Thermodynamics, (Sausalito, Calif., University Science Books, 1999) pp. 1-656.

14) W. J. Hehre, L. Radom, P. v. R. Schleyer and J. A. Pople: AB initio Molecular Orbital Theory, (New York: Wiley. 1986) pp. 226-261.

15) J. E. Lee, W. Choi and B. J. Mhin: J. Phys. Chem. A 107 (2003) 26932699.

16) A. Yazawa, S. Nakazawa and N. Menad: Chemosphere 39 (1999) 2419-2432.

17) N. Menad, B. Bjorkman, S. Zhang and E. Forsberg: EPD Congress 1998, ed. by B. Mishra, (TMS, 1998) pp. 657-673.

18) N. Menad and B. Bjorkman: report No. 5, Jan. 1997, 25pp. Div. of Process Metallurgy, Lulea University of Technology, Sweden.

19) E. Shibata, S. Yamamoto, E. Kasai and T. Nakamura: Chemosphere 50 (2003) $1235-1242$

\section{Appendix}

Table A.1 Evaluated thermodynamic properties of gaseous PXDDs from $298.15 \mathrm{~K}$ to $1500 \mathrm{~K}$ at $101.325 \mathrm{kPa}$.

\begin{tabular}{|c|c|c|c|c|c|c|c|}
\hline \multirow{3}{*}{ Compound } & \multirow{3}{*}{$\begin{array}{c}\Delta_{\mathrm{f}} H^{\circ}(298 \mathrm{~K}) \\
\mathrm{kJ} \cdot \mathrm{mol}^{-1}\end{array}$} & \multirow{3}{*}{$\begin{array}{c}\Delta_{\mathrm{f}} G^{\circ}(298 \mathrm{~K}) \\
\mathrm{kJ} \cdot \mathrm{mol}^{-1}\end{array}$} & \multirow{3}{*}{$\begin{array}{c}S^{\circ}(298 \mathrm{~K}) \\
\mathrm{J} \cdot \mathrm{mol}^{-1} \cdot \mathrm{K}^{-1}\end{array}$} & \multirow{2}{*}{\multicolumn{4}{|c|}{$\begin{array}{c}C_{\mathrm{p}}, \mathrm{J} \cdot \mathrm{mol}^{-1} \cdot \mathrm{K}^{-1} \\
C_{\mathrm{p}}=\mathrm{A} T^{3}+\mathrm{B} T^{2}+\mathrm{C} T+\mathrm{D}\end{array}$}} \\
\hline & & & & & & & \\
\hline & & & & $\mathrm{A} \times 10^{7}$ & $\mathrm{~B} \times 10^{4}$ & $\mathrm{C} \times 10$ & $\mathrm{D}$ \\
\hline $1-\mathrm{Br}_{1}-2-\mathrm{Cl}_{1}-\mathrm{DD}$ & -45.0 & 66.8 & 479.3 & 1.682 & -6.539 & 9.270 & -6.906 \\
\hline $1-\mathrm{Br}_{1}-3-\mathrm{Cl}_{1}-\mathrm{DD}$ & -53.1 & 57.9 & 481.8 & 1.679 & -6.526 & 9.251 & -6.022 \\
\hline $1-\mathrm{Br}_{1}-4-\mathrm{Cl}_{1}-\mathrm{DD}$ & -46.4 & 64.6 & 482.1 & 1.676 & -6.520 & 9.252 & -6.533 \\
\hline $1-\mathrm{Br}_{1}-6-\mathrm{Cl}_{1}-\mathrm{DD}$ & -47.9 & 63.2 & 481.5 & 1.677 & -6.523 & 9.254 & -6.567 \\
\hline $1-\mathrm{Br}_{1}-7-\mathrm{Cl}_{1}-\mathrm{DD}$ & -55.4 & 55.9 & 480.9 & 1.681 & -6.532 & 9.256 & -6.193 \\
\hline $1-\mathrm{Br}_{1}-8-\mathrm{Cl}_{1}-\mathrm{DD}$ & -55.2 & 56.2 & 480.9 & 1.681 & -6.532 & 9.257 & -6.210 \\
\hline $1-\mathrm{Br}_{1}-9-\mathrm{Cl}_{1}-\mathrm{DD}$ & -46.4 & 65.1 & 480.3 & 1.678 & -6.526 & 9.257 & -6.656 \\
\hline $2-\mathrm{Br}_{1}-1-\mathrm{Cl}_{1}-\mathrm{DD}$ & -43.4 & 68.1 & 480.2 & 1.679 & -6.529 & 9.259 & -6.534 \\
\hline $2-\mathrm{Br}_{1}-3-\mathrm{Cl}_{1}-\mathrm{DD}$ & -50.6 & 60.9 & 480.3 & 1.682 & -6.534 & 9.258 & -6.083 \\
\hline $2-\mathrm{Br}_{1}-4-\mathrm{Cl}_{1}-\mathrm{DD}$ & -51.3 & 59.7 & 482.0 & 1.676 & -6.516 & 9.240 & -5.583 \\
\hline $2-\mathrm{Br}_{1}-7-\mathrm{Cl}_{1}-\mathrm{DD}$ & -61.1 & 50.4 & 480.5 & 1.682 & -6.533 & 9.250 & -5.530 \\
\hline $2-\mathrm{Br}_{1}-8-\mathrm{Cl}_{1}-\mathrm{DD}$ & -60.9 & 50.4 & 481.0 & 1.682 & -6.532 & 9.249 & -5.414 \\
\hline $2-\mathrm{Br}_{1}-9-\mathrm{Cl}_{1}-\mathrm{DD}$ & -53.2 & 57.8 & 481.9 & 1.678 & -6.523 & 9.245 & -5.683 \\
\hline $1-\mathrm{Br}_{1}-2,3-\mathrm{Cl}_{2}-\mathrm{DD}$ & -58.6 & 58.5 & 508.2 & 1.691 & -6.528 & 9.134 & 13.13 \\
\hline $1-\mathrm{Br}_{1}-2,4-\mathrm{Cl}_{2}-\mathrm{DD}$ & -62.0 & 54.4 & 510.3 & 1.685 & -6.510 & 9.118 & 13.50 \\
\hline $1-\mathrm{Br}_{1}-2,6-\mathrm{Cl}_{2}-\mathrm{DD}$ & -65.7 & 50.6 & 510.8 & 1.689 & -6.519 & 9.123 & 13.52 \\
\hline $1-\mathrm{Br}_{1}-2,7-\mathrm{Cl}_{2}-\mathrm{DD}$ & -73.1 & 43.5 & 509.7 & 1.693 & -6.529 & 9.128 & 13.76 \\
\hline $1-\mathrm{Br}_{1}-2,8-\mathrm{Cl}_{2}-\mathrm{DD}$ & -72.9 & 43.5 & 510.5 & 1.693 & -6.529 & 9.127 & 13.83 \\
\hline $1-\mathrm{Br}_{1}-2,9-\mathrm{Cl}_{2}-\mathrm{DD}$ & -64.2 & 52.5 & 509.5 & 1.690 & -6.523 & 9.128 & 13.31 \\
\hline $1-\mathrm{Br}_{1}-3,4-\mathrm{Cl}_{2}-\mathrm{DD}$ & -60.7 & 55.7 & 510.6 & 1.684 & -6.507 & 9.117 & 13.37 \\
\hline $1-\mathrm{Br}_{1}-3,6-\mathrm{Cl}_{2}-\mathrm{DD}$ & -73.4 & 42.4 & 512.6 & 1.685 & -6.506 & 9.107 & 14.17 \\
\hline $1-\mathrm{Br}_{1}-3,7-\mathrm{Cl}_{2}-\mathrm{DD}$ & -81.0 & 35.0 & 511.9 & 1.689 & -6.515 & 9.109 & 14.60 \\
\hline $1-\mathrm{Br}_{1}-4,6-\mathrm{Cl}_{2}-\mathrm{DD}$ & -65.5 & 50.9 & 510.4 & 1.683 & -6.502 & 9.110 & 13.60 \\
\hline $1-\mathrm{Br}_{1}-4,7-\mathrm{Cl}_{2}-\mathrm{DD}$ & -74.3 & 41.8 & 511.7 & 1.686 & -6.510 & 9.111 & 14.05 \\
\hline $1-\mathrm{Br}_{1}-4,8-\mathrm{Cl}_{2}-\mathrm{DD}$ & -74.3 & 41.9 & 511.2 & 1.686 & -6.510 & 9.112 & 13.95 \\
\hline $1-\mathrm{Br}_{1}-4,9-\mathrm{Cl}_{2}-\mathrm{DD}$ & -65.6 & 50.6 & 511.2 & 1.682 & -6.500 & 9.109 & 13.60 \\
\hline $1-\mathrm{Br}_{1}-6,7-\mathrm{Cl}_{2}-\mathrm{DD}$ & -64.2 & 52.2 & 510.7 & 1.686 & -6.513 & 9.120 & 13.42 \\
\hline $1-\mathrm{Br}_{1}-6,8-\mathrm{Cl}_{2}-\mathrm{DD}$ & -73.0 & 43.1 & 511.5 & 1.685 & -6.505 & 9.106 & 14.14 \\
\hline $1-\mathrm{Br}_{1}-6,9-\mathrm{Cl}_{2}-\mathrm{DD}$ & -65.4 & 50.6 & 511.7 & 1.681 & -6.496 & 9.104 & 13.79 \\
\hline $1-\mathrm{Br}_{1}-7,8-\mathrm{Cl}_{2}-\mathrm{DD}$ & -71.1 & 45.6 & 509.8 & 1.689 & -6.519 & 9.120 & 13.37 \\
\hline $1-\mathrm{Br}_{1}-7,9-\mathrm{Cl}_{2}-\mathrm{DD}$ & -71.8 & 44.1 & 512.2 & 1.685 & -6.506 & 9.106 & 14.21 \\
\hline
\end{tabular}




\begin{tabular}{|c|c|c|c|c|c|c|c|}
\hline \multirow{2}{*}{ Compound } & \multirow{2}{*}{$\begin{array}{c}\Delta_{\mathrm{f}} H^{\circ}(298 \mathrm{~K}) \\
\mathrm{kJ} \cdot \mathrm{mol}^{-1}\end{array}$} & \multirow{2}{*}{$\begin{array}{c}\Delta_{\mathrm{f}} G^{\circ}(298 \mathrm{~K}) \\
\mathrm{kJ} \cdot \mathrm{mol}^{-1}\end{array}$} & \multirow{2}{*}{$\begin{array}{l}S^{\circ}(298 \mathrm{~K}) \\
\mathrm{J} \cdot \mathrm{mol}^{-1} \cdot \mathrm{K}^{-1}\end{array}$} & \multicolumn{4}{|c|}{$\begin{array}{c}C_{\mathrm{p}}, \mathrm{J} \cdot \mathrm{mol}^{-1} \cdot \mathrm{K}^{-1} \\
C_{\mathrm{p}}=\mathrm{A} T^{3}+\mathrm{B} T^{2}+\mathrm{C} T+\mathrm{D}\end{array}$} \\
\hline & & & & $\mathrm{A} \times 10^{7}$ & $\mathrm{~B} \times 10^{4}$ & $\mathrm{C} \times 10$ & D \\
\hline $1-\mathrm{Br}_{1}-8,9-\mathrm{Cl}_{2}-\mathrm{DD}$ & -62.6 & 54.0 & 509.9 & 1.687 & -6.517 & 9.124 & 13.28 \\
\hline $2-\mathrm{Br}_{1}-1,3-\mathrm{Cl}_{2}-\mathrm{DD}$ & -58.3 & 58.8 & 508.2 & 1.690 & -6.523 & 9.128 & 13.40 \\
\hline $2-\mathrm{Br}_{1}-1,4-\mathrm{Cl}_{2}-\mathrm{DD}$ & -60.6 & 56.1 & 509.5 & 1.682 & -6.500 & 9.108 & 13.76 \\
\hline $2-\mathrm{Br}_{1}-1,6-\mathrm{Cl}_{2}-\mathrm{DD}$ & -64.0 & 52.4 & 510.5 & 1.686 & -6.511 & 9.116 & 13.67 \\
\hline $2-\mathrm{Br}_{1}-1,7-\mathrm{Cl}_{2}-\mathrm{DD}$ & -71.4 & 45.0 & 510.4 & 1.690 & -6.521 & 9.119 & 14.04 \\
\hline $2-\mathrm{Br}_{1}-1,8-\mathrm{Cl}_{2}-\mathrm{DD}$ & -71.2 & 45.2 & 510.7 & 1.690 & -6.520 & 9.118 & 14.10 \\
\hline $2-\mathrm{Br}_{1}-1,9-\mathrm{Cl}_{2}-\mathrm{DD}$ & -62.3 & 54.3 & 509.9 & 1.687 & -6.513 & 9.119 & 13.56 \\
\hline $2-\mathrm{Br}_{1}-3,4-\mathrm{Cl}_{2}-\mathrm{DD}$ & -57.0 & 60.3 & 507.7 & 1.689 & -6.521 & 9.127 & 13.37 \\
\hline $2-\mathrm{Br}_{1}-3,6-\mathrm{Cl}_{2}-\mathrm{DD}$ & -71.0 & 45.5 & 510.1 & 1.689 & -6.517 & 9.117 & 14.04 \\
\hline $2-\mathrm{Br}_{1}-3,7-\mathrm{Cl}_{2}-\mathrm{DD}$ & -78.4 & 38.0 & 510.6 & 1.692 & -6.525 & 9.118 & 14.49 \\
\hline $2-\mathrm{Br}_{1}-3,8-\mathrm{Cl}_{2}-\mathrm{DD}$ & -78.6 & 37.9 & 510.4 & 1.692 & -6.524 & 9.117 & 14.54 \\
\hline $2-\mathrm{Br}_{1}-3,9-\mathrm{Cl}_{2}-\mathrm{DD}$ & -71.0 & 46.0 & 508.7 & 1.689 & -6.519 & 9.119 & 13.92 \\
\hline $2-\mathrm{Br}_{1}-4,6-\mathrm{Cl}_{2}-\mathrm{DD}$ & -70.0 & 45.8 & 512.5 & 1.683 & -6.499 & 9.098 & 14.66 \\
\hline $2-\mathrm{Br}_{1}-4,7-\mathrm{Cl}_{2}-\mathrm{DD}$ & -78.9 & 37.2 & 511.7 & 1.687 & -6.506 & 9.099 & 14.96 \\
\hline $2-\mathrm{Br}_{1}-4,8-\mathrm{Cl}_{2}-\mathrm{DD}$ & -79.0 & 36.7 & 512.8 & 1.686 & -6.506 & 9.098 & 15.08 \\
\hline $2-\mathrm{Br}_{1}-4,9-\mathrm{Cl}_{2}-\mathrm{DD}$ & -71.6 & 44.4 & 512.1 & 1.683 & -6.497 & 9.096 & 14.64 \\
\hline $2-\mathrm{Br}_{1}-6,7-\mathrm{Cl}_{2}-\mathrm{DD}$ & -69.5 & 46.5 & 511.9 & 1.688 & -6.514 & 9.112 & 14.28 \\
\hline $2-\mathrm{Br}_{1}-6,8-\mathrm{Cl}_{2}-\mathrm{DD}$ & -78.7 & 37.3 & 511.9 & 1.686 & -6.504 & 9.097 & 15.01 \\
\hline $2-\mathrm{Br}_{1}-6,9-\mathrm{Cl}_{2}-\mathrm{DD}$ & -72.1 & 43.7 & 512.6 & 1.674 & -6.470 & 9.071 & 15.37 \\
\hline 2- $\mathrm{Br}_{1}-7,8-\mathrm{Cl}_{2}-\mathrm{DD}$ & -76.7 & 39.5 & 511.3 & 1.690 & -6.517 & 9.111 & 14.65 \\
\hline $2-\mathrm{Br}_{1}-7,9-\mathrm{Cl}_{2}-\mathrm{DD}$ & -78.5 & 37.5 & 511.7 & 1.686 & -6.505 & 9.099 & 14.95 \\
\hline $2-\mathrm{Br}_{1}-8,9-\mathrm{Cl}_{2}-\mathrm{DD}$ & -69.2 & 47.2 & 510.5 & 1.688 & -6.514 & 9.113 & 14.19 \\
\hline $1-\mathrm{Br}_{1}-2,3,4-\mathrm{Cl}_{3}-\mathrm{DD}$ & -63.1 & 59.5 & 536.6 & 1.698 & -6.514 & 9.003 & 32.55 \\
\hline $1-\mathrm{Br}_{1}-2,3,7-\mathrm{Cl}_{3}-\mathrm{DD}$ & -85.9 & 36.0 & 538.5 & 1.702 & -6.520 & 8.995 & 33.72 \\
\hline $1-\mathrm{Br}_{1}-2,3,9-\mathrm{Cl}_{3}-\mathrm{DD}$ & -77.2 & 44.6 & 539.1 & 1.699 & -6.512 & 8.993 & 33.38 \\
\hline $1-\mathrm{Br}_{1}-2,4,6-\mathrm{Cl}_{3}-\mathrm{DD}$ & -80.6 & 40.8 & 540.2 & 1.692 & -6.492 & 8.975 & 33.68 \\
\hline $1-\mathrm{Br}_{1}-2,4,8-\mathrm{Cl}_{3}-\mathrm{DD}$ & -89.2 & 32.1 & 540.7 & 1.696 & -6.501 & 8.978 & 34.07 \\
\hline $1-\mathrm{Br}_{1}-2,6,7-\mathrm{Cl}_{3}-\mathrm{DD}$ & -81.4 & 40.2 & 539.7 & 1.699 & -6.511 & 8.992 & 33.35 \\
\hline $1-\mathrm{Br}_{1}-2,6,9-\mathrm{Cl}_{3}-\mathrm{DD}$ & -82.7 & 38.5 & 541.1 & 1.693 & -6.495 & 8.976 & 33.77 \\
\hline $1-\mathrm{Br}_{1}-2,7,8-\mathrm{Cl}_{3}-\mathrm{DD}$ & -88.3 & 33.6 & 538.5 & 1.702 & -6.518 & 8.993 & 33.66 \\
\hline $1-\mathrm{Br}_{1}-2,8,9-\mathrm{Cl}_{3}-\mathrm{DD}$ & -79.9 & 42.2 & 538.1 & 1.700 & -6.515 & 8.996 & 33.18 \\
\hline $1-\mathrm{Br}_{1}-3,4,8-\mathrm{Cl}_{3}-\mathrm{DD}$ & -87.8 & 33.4 & 541.0 & 1.695 & -6.498 & 8.978 & 33.85 \\
\hline $1-\mathrm{Br}_{1}-3,4,9-\mathrm{Cl}_{3}-\mathrm{DD}$ & -79.4 & 41.7 & 541.4 & 1.691 & -6.489 & 8.974 & 33.57 \\
\hline $1-\mathrm{Br}_{1}-3,6,8-\mathrm{Cl}_{3}-\mathrm{DD}$ & -97.7 & 22.9 & 542.9 & 1.693 & -6.489 & 8.960 & 34.92 \\
\hline $1-\mathrm{Br}_{1}-3,7,9-\mathrm{Cl}_{3}-\mathrm{DD}$ & -96.8 & 24.2 & 541.8 & 1.694 & -6.491 & 8.963 & 34.77 \\
\hline $1-\mathrm{Br}_{1}-4,6,7-\mathrm{Cl}_{3}-\mathrm{DD}$ & -81.0 & 40.3 & 540.6 & 1.692 & -6.493 & 8.978 & 33.51 \\
\hline $1-\mathrm{Br}_{1}-4,7,8-\mathrm{Cl}_{3}-\mathrm{DD}$ & -89.3 & 31.9 & 540.9 & 1.695 & -6.497 & 8.976 & 33.95 \\
\hline $1-\mathrm{Br}_{1}-6,7,8-\mathrm{Cl}_{3}-\mathrm{DD}$ & -76.5 & 45.1 & 539.6 & 1.695 & -6.502 & 8.987 & 33.27 \\
\hline $1-\mathrm{Br}_{1}-6,7,9-\mathrm{Cl}_{3}-\mathrm{DD}$ & -78.8 & 42.4 & 540.7 & 1.689 & -6.482 & 8.968 & 33.68 \\
\hline $2-\mathrm{Br}_{1}-1,3,4-\mathrm{Cl}_{3}-\mathrm{DD}$ & -62.6 & 59.3 & 538.6 & 1.696 & -6.506 & 8.994 & 32.99 \\
\hline $2-\mathrm{Br}_{1}-1,3,6-\mathrm{Cl}_{3}-\mathrm{DD}$ & -78.2 & 43.9 & 538.2 & 1.697 & -6.505 & 8.986 & 33.64 \\
\hline $2-\mathrm{Br}_{1}-1,3,8-\mathrm{Cl}_{3}-\mathrm{DD}$ & -85.5 & 36.3 & 539.1 & 1.701 & -6.515 & 8.989 & 33.99 \\
\hline $2-\mathrm{Br}_{1}-1,4,6-\mathrm{Cl}_{3}-\mathrm{DD}$ & -79.1 & 42.1 & 541.0 & 1.689 & -6.480 & 8.963 & 34.06 \\
\hline $2-\mathrm{Br}_{1}-1,4,7-\mathrm{Cl}_{3}-\mathrm{DD}$ & -87.6 & 33.5 & 541.3 & 1.693 & -6.490 & 8.966 & 34.45 \\
\hline $2-\mathrm{Br}_{1}-1,4,9-\mathrm{Cl}_{3}-\mathrm{DD}$ & -79.0 & 42.2 & 540.9 & 1.702 & -6.522 & 9.005 & 32.73 \\
\hline $2-\mathrm{Br}_{1}-1,6,8-\mathrm{Cl}_{3}-\mathrm{DD}$ & -88.6 & 32.5 & 541.5 & 1.694 & -6.493 & 8.968 & 34.44 \\
\hline $2-\mathrm{Br}_{1}-, 1,7,8-\mathrm{Cl}_{3}-\mathrm{DD}$ & -86.5 & 35.1 & 539.6 & 1.699 & -6.508 & 8.984 & 33.99 \\
\hline $2-\mathrm{Br}_{1}-1,8,9-\mathrm{Cl}_{3}-\mathrm{DD}$ & -77.9 & 43.4 & 540.8 & 1.697 & -6.505 & 8.985 & 33.64 \\
\hline $2-\mathrm{Br}_{1}-3,4,7-\mathrm{Cl}_{3}-\mathrm{DD}$ & -83.9 & 37.9 & 539.0 & 1.700 & -6.512 & 8.987 & 33.94 \\
\hline $2-\mathrm{Br}_{1}-3,4,9-\mathrm{Cl}_{3}-\mathrm{DD}$ & -76.7 & 45.1 & 538.9 & 1.695 & -6.501 & 8.982 & 33.65 \\
\hline $2-\mathrm{Br}_{1}-3,6,7-\mathrm{Cl}_{3}-\mathrm{DD}$ & -86.5 & 35.3 & 539.0 & 1.699 & -6.510 & 8.985 & 33.96 \\
\hline $2-\mathrm{Br}_{1}-3,6,9-\mathrm{Cl}_{3}-\mathrm{DD}$ & -89.1 & 32.2 & 540.6 & 1.693 & -6.491 & 8.967 & 34.43 \\
\hline $2-\mathrm{Br}_{1}-3,7,8-\mathrm{Cl}_{3}-\mathrm{DD}$ & -93.8 & 27.9 & 539.4 & 1.701 & -6.513 & 8.982 & 34.43 \\
\hline $2-\mathrm{Br}_{1}-3,8,9-\mathrm{Cl}_{3}-\mathrm{DD}$ & -86.4 & 35.4 & 538.9 & 1.699 & -6.509 & 8.985 & 33.96 \\
\hline $2-\mathrm{Br}_{1}-4,6,8-\mathrm{Cl}_{3}-\mathrm{DD}$ & -94.8 & 26.4 & 540.9 & 1.692 & -6.484 & 8.955 & 35.11 \\
\hline
\end{tabular}




\begin{tabular}{|c|c|c|c|c|c|c|c|}
\hline \multirow[t]{2}{*}{ Compound } & \multirow{2}{*}{$\begin{array}{c}\Delta_{\mathrm{f}} H^{\circ}(298 \mathrm{~K}) \\
\mathrm{kJ} \cdot \mathrm{mol}^{-1}\end{array}$} & \multirow{2}{*}{$\begin{array}{c}\Delta_{\mathrm{f}} G^{\circ}(298 \mathrm{~K}) \\
\mathrm{kJ} \cdot \mathrm{mol}^{-1}\end{array}$} & \multirow{2}{*}{$\begin{array}{c}S^{\circ}(298 \mathrm{~K}) \\
\mathrm{J} \cdot \mathrm{mol}^{-1} \cdot \mathrm{K}^{-1}\end{array}$} & \multicolumn{4}{|c|}{$\begin{array}{c}C_{\mathrm{p}}, \mathrm{J} \cdot \mathrm{mol}^{-1} \cdot \mathrm{K}^{-1} \\
C_{\mathrm{p}}=\mathrm{A} T^{3}+\mathrm{B} T^{2}+\mathrm{C} T+\mathrm{D}\end{array}$} \\
\hline & & & & $\mathrm{A} \times 10^{7}$ & $\mathrm{~B} \times 10^{4}$ & $\mathrm{C} \times 10$ & $\mathrm{D}$ \\
\hline $2-\mathrm{Br}_{1}-4,7,8-\mathrm{Cl}_{3}-\mathrm{DD}$ & -94.0 & 27.0 & 541.9 & 1.695 & -6.494 & 8.964 & 34.97 \\
\hline $2-\mathrm{Br}_{1}-4,8,9-\mathrm{Cl}_{3}-\mathrm{DD}$ & -87.0 & 34.1 & 541.5 & 1.693 & -6.489 & 8.965 & 34.51 \\
\hline $2-\mathrm{Br}_{1}-6,7,8-\mathrm{Cl}_{3}-\mathrm{DD}$ & -82.1 & 39.5 & 539.5 & 1.697 & -6.505 & 8.978 & 34.16 \\
\hline $2-\mathrm{Br}_{1}-6,8,9-\mathrm{Cl}_{3}-\mathrm{DD}$ & -85.3 & 35.5 & 542.1 & 1.690 & -6.482 & 8.958 & 34.66 \\
\hline $1-\mathrm{Br}_{1}-2,3,4,6-\mathrm{Cl}_{4}-\mathrm{DD}$ & -81.0 & 46.1 & 567.8 & 1.705 & -6.496 & 8.862 & 52.76 \\
\hline $1-\mathrm{Br}_{1}-2,3,4,8-\mathrm{Cl}_{4}-\mathrm{DD}$ & -89.7 & 37.7 & 566.9 & 1.709 & -6.506 & 8.865 & 53.02 \\
\hline $1-\mathrm{Br}_{1}-2,3,6,8-\mathrm{Cl}_{4}-\mathrm{DD}$ & -102.5 & 24.1 & 569.5 & 1.706 & -6.494 & 8.845 & 54.10 \\
\hline $1-\mathrm{Br}_{1}-2,3,7,8-\mathrm{Cl}_{4}-\mathrm{DD}$ & -100.6 & 26.5 & 567.6 & 1.712 & -6.510 & 8.862 & 53.64 \\
\hline $1-\mathrm{Br}_{1}-2,3,8,9-\mathrm{Cl}_{4}-\mathrm{DD}$ & -92.4 & 34.7 & 567.7 & 1.709 & -6.504 & 8.862 & 53.26 \\
\hline $1-\mathrm{Br}_{1}-2,4,6,8-\mathrm{Cl}_{4}-\mathrm{DD}$ & -104.6 & 21.6 & 570.9 & 1.700 & -6.474 & 8.828 & 54.40 \\
\hline $1-\mathrm{Br}_{1}-2,4,7,8-\mathrm{Cl}_{4}-\mathrm{DD}$ & -103.9 & 22.8 & 569.2 & 1.705 & -6.491 & 8.845 & 53.94 \\
\hline $1-\mathrm{Br}_{1}-2,4,8,9-\mathrm{Cl}_{4}-\mathrm{DD}$ & -95.7 & 30.5 & 570.7 & 1.702 & -6.484 & 8.844 & 53.55 \\
\hline $1-\mathrm{Br}_{1}-2,6,7,9-\mathrm{Cl}_{4}-\mathrm{DD}$ & -95.8 & 30.9 & 569.2 & 1.701 & -6.481 & 8.841 & 53.64 \\
\hline $1-\mathrm{Br}_{1}-2,7,8,9-\mathrm{Cl}_{4}-\mathrm{DD}$ & -92.2 & 34.8 & 568.4 & 1.708 & -6.503 & 8.860 & 53.27 \\
\hline $1-\mathrm{Br}_{1}-3,4,6,8-\mathrm{Cl}_{4}-\mathrm{DD}$ & -103.0 & 23.1 & 570.9 & 1.699 & -6.473 & 8.829 & 54.23 \\
\hline $1-\mathrm{Br}_{1}-3,4,7,8-\mathrm{Cl}_{4}-\mathrm{DD}$ & -102.5 & 24.1 & 569.4 & 1.704 & -6.487 & 8.843 & 53.79 \\
\hline $1-\mathrm{Br}_{1}-3,4,8,9-\mathrm{Cl}_{4}-\mathrm{DD}$ & -94.5 & 31.9 & 570.4 & 1.701 & -6.482 & 8.843 & 53.41 \\
\hline $1-\mathrm{Br}_{1}-3,6,7,9-\mathrm{Cl}_{4}-\mathrm{DD}$ & -103.1 & 23.1 & 570.9 & 1.697 & -6.467 & 8.824 & 54.37 \\
\hline $1-\mathrm{Br}_{1}-3,7,8,9-\mathrm{Cl}_{4}-\mathrm{DD}$ & -99.9 & 26.8 & 569.4 & 1.705 & -6.491 & 8.845 & 53.85 \\
\hline $1-\mathrm{Br}_{1}-4,6,7,9-\mathrm{Cl}_{4}-\mathrm{DD}$ & -95.2 & 30.9 & 571.2 & 1.694 & -6.461 & 8.824 & 53.89 \\
\hline $1-\mathrm{Br}_{1}-4,7,8,9-\mathrm{Cl}_{4}-\mathrm{DD}$ & -93.2 & 33.3 & 569.9 & 1.701 & -6.483 & 8.843 & 53.48 \\
\hline $1-\mathrm{Br}_{1}-6,7,8,9-\mathrm{Cl}_{4}-\mathrm{DD}$ & -79.2 & 47.8 & 568.2 & 1.700 & -6.484 & 8.853 & 52.76 \\
\hline $2-\mathrm{Br}_{1}-1,3,4,7-\mathrm{Cl}_{4}-\mathrm{DD}$ & -89.3 & 38.0 & 567.4 & 1.707 & -6.500 & 8.857 & 53.43 \\
\hline $2-\mathrm{Br}_{1}-1,3,4,9-\mathrm{Cl}_{4}-\mathrm{DD}$ & -80.7 & 46.3 & 568.4 & 1.703 & -6.489 & 8.852 & 53.20 \\
\hline $2-\mathrm{Br}_{1}-1,3,6,7-\mathrm{Cl}_{4}-\mathrm{DD}$ & -93.3 & 33.7 & 568.1 & 1.707 & -6.499 & 8.855 & 53.49 \\
\hline $2-\mathrm{Br}_{1}-1,3,6,9-\mathrm{Cl}_{4}-\mathrm{DD}$ & -94.4 & 32.4 & 568.9 & 1.702 & -6.483 & 8.841 & 53.84 \\
\hline $2-\mathrm{Br}_{1}-1,3,7,8-\mathrm{Cl}_{4}-\mathrm{DD}$ & -100.3 & 27.1 & 567.0 & 1.710 & -6.505 & 8.856 & 53.85 \\
\hline $2-\mathrm{Br}_{1}-1,4,6,7-\mathrm{Cl}_{4}-\mathrm{DD}$ & -94.2 & 32.1 & 570.5 & 1.699 & -6.474 & 8.833 & 53.94 \\
\hline $2-\mathrm{Br}_{1}-1,4,6,9-\mathrm{Cl}_{4}-\mathrm{DD}$ & -95.3 & 30.8 & 571.1 & 1.693 & -6.456 & 8.816 & 54.30 \\
\hline $2-\mathrm{Br}_{1}-1,4,7,9-\mathrm{Cl}_{4}-\mathrm{DD}$ & -102.8 & 23.4 & 570.9 & 1.697 & -6.465 & 8.819 & 54.67 \\
\hline $2-\mathrm{Br}_{1}-1,6,7,8-\mathrm{Cl}_{4}-\mathrm{DD}$ & -91.8 & 35.1 & 568.6 & 1.705 & -6.492 & 8.850 & 53.51 \\
\hline $2-\mathrm{Br}_{1}-1,6,8,9-\mathrm{Cl}_{4}-\mathrm{DD}$ & -93.7 & 32.7 & 570.4 & 1.698 & -6.472 & 8.831 & 53.96 \\
\hline $2-\mathrm{Br}_{1}-3,4,6,8-\mathrm{Cl}_{4}-\mathrm{DD}$ & -99.5 & 27.3 & 569.0 & 1.704 & -6.487 & 8.839 & 54.29 \\
\hline $2-\mathrm{Br}_{1}-3,4,7,8-\mathrm{Cl}_{4}-\mathrm{DD}$ & -98.9 & 28.0 & 568.3 & 1.709 & -6.501 & 8.852 & 53.93 \\
\hline $2-\mathrm{Br}_{1}-3,4,8,9-\mathrm{Cl}_{4}-\mathrm{DD}$ & -92.0 & 34.9 & 568.6 & 1.706 & -6.494 & 8.851 & 53.59 \\
\hline $2-\mathrm{Br}_{1}-3,6,7,8-\mathrm{Cl}_{4}-\mathrm{DD}$ & -98.6 & 28.3 & 568.3 & 1.708 & -6.499 & 8.850 & 53.95 \\
\hline $2-\mathrm{Br}_{1}-3,7,8,9-\mathrm{Cl}_{4}-\mathrm{DD}$ & -98.7 & 28.5 & 567.5 & 1.708 & -6.499 & 8.852 & 53.83 \\
\hline $2-\mathrm{Br}_{1}-4,6,7,8-\mathrm{Cl}_{4}-\mathrm{DD}$ & -97.7 & 28.9 & 569.6 & 1.703 & -6.483 & 8.835 & 54.30 \\
\hline $2-\mathrm{Br}_{1}-4,6,8,9-\mathrm{Cl}_{4}-\mathrm{DD}$ & -101.0 & 25.1 & 571.3 & 1.695 & -6.458 & 8.812 & 54.87 \\
\hline $2-\mathrm{Br}_{1}-6,7,8,9-\mathrm{Cl}_{4}-\mathrm{DD}$ & -85.7 & 41.3 & 568.1 & 1.703 & -6.486 & 8.847 & 53.53 \\
\hline $1-\mathrm{Br}_{1}-2,3,4,6,8-\mathrm{Cl}_{5}-\mathrm{DD}$ & -104.7 & 27.1 & 598.7 & 1.713 & -6.480 & 8.716 & 73.43 \\
\hline $1-\mathrm{Br}_{1}-2,3,4,7,8-\mathrm{Cl}_{5}-\mathrm{DD}$ & -104.0 & 28.6 & 596.0 & 1.718 & -6.496 & 8.732 & 72.90 \\
\hline $1-\mathrm{Br}_{1}-2,3,4,8,9-\mathrm{Cl}_{5}-\mathrm{DD}$ & -96.1 & 36.2 & 596.8 & 1.716 & -6.491 & 8.732 & 72.57 \\
\hline $1-\mathrm{Br}_{1}-2,3,6,7,8-\mathrm{Cl}_{5}-\mathrm{DD}$ & -105.3 & 27.0 & 596.8 & 1.717 & -6.493 & 8.727 & 73.21 \\
\hline $1-\mathrm{Br}_{1}-2,3,6,7,9-\mathrm{Cl}_{5}-\mathrm{DD}$ & -107.6 & 24.3 & 598.4 & 1.711 & -6.472 & 8.708 & 73.62 \\
\hline $1-\mathrm{Br}_{1}-2,3,7,8,9-\mathrm{Cl}_{5}-\mathrm{DD}$ & -104.3 & 28.2 & 596.1 & 1.718 & -6.495 & 8.730 & 73.05 \\
\hline $1-\mathrm{Br}_{1}-2,4,6,7,9-\mathrm{Cl}_{5}-\mathrm{DD}$ & -109.6 & 21.6 & 600.5 & 1.704 & -6.453 & 8.692 & 73.91 \\
\hline $1-\mathrm{Br}_{1}-2,4,7,8,9-\mathrm{Cl}_{5}-\mathrm{DD}$ & -107.5 & 24.6 & 597.5 & 1.712 & -6.476 & 8.712 & 73.40 \\
\hline $1-\mathrm{Br}_{1}-3,4,6,7,8-\mathrm{Cl}_{5}-\mathrm{DD}$ & -105.9 & 26.1 & 597.8 & 1.710 & -6.473 & 8.712 & 73.26 \\
\hline $1-\mathrm{Br}_{1}-3,4,6,8,9-\mathrm{Cl}_{5}-\mathrm{DD}$ & -108.1 & 22.9 & 601.2 & 1.703 & -6.450 & 8.690 & 73.79 \\
\hline $1-\mathrm{Br}_{1}-3,6,7,8,9-\mathrm{Cl}_{5}-\mathrm{DD}$ & -103.1 & 28.7 & 598.6 & 1.710 & -6.472 & 8.710 & 73.38 \\
\hline $1-\mathrm{Br}_{1}-4,6,7,8,9-\mathrm{Cl}_{5}-\mathrm{DD}$ & -95.3 & 36.3 & 599.3 & 1.707 & -6.465 & 8.710 & 72.86 \\
\hline 2- $\mathrm{Br}_{1}-1,3,4,6,7-\mathrm{Cl}_{5}-\mathrm{DD}$ & -95.6 & 37.0 & 596.1 & 1.714 & -6.484 & 8.725 & 72.90 \\
\hline 2- $\mathrm{Br}_{1}-1,3,4,6,9-\mathrm{Cl}_{5}-\mathrm{DD}$ & -96.7 & 35.2 & 598.3 & 1.707 & -6.465 & 8.705 & 73.44 \\
\hline $2-\mathrm{Br}_{1}-1,3,4,7,8-\mathrm{Cl}_{5}-\mathrm{DD}$ & -103.7 & 28.8 & 596.1 & 1.717 & -6.490 & 8.725 & 73.31 \\
\hline
\end{tabular}




\begin{tabular}{|c|c|c|c|c|c|c|c|}
\hline \multirow{2}{*}{ Compound } & \multirow{2}{*}{$\begin{array}{c}\Delta_{\mathrm{f}} H^{\circ}(298 \mathrm{~K}) \\
\mathrm{kJ} \cdot \mathrm{mol}^{-1}\end{array}$} & \multirow{2}{*}{$\begin{array}{c}\Delta_{\mathrm{f}} G^{\circ}(298 \mathrm{~K}) \\
\mathrm{kJ} \cdot \mathrm{mol}^{-1}\end{array}$} & \multirow{2}{*}{$\begin{array}{c}S^{\circ}(298 \mathrm{~K}) \\
\mathrm{J} \cdot \mathrm{mol}^{-1} \cdot \mathrm{K}^{-1}\end{array}$} & \multicolumn{4}{|c|}{$\begin{array}{c}C_{\mathrm{p}}, \mathrm{J} \cdot \mathrm{mol}^{-1} \cdot \mathrm{K}^{-1} \\
C_{\mathrm{p}}=\mathrm{A} T^{3}+\mathrm{B} T^{2}+\mathrm{C} T+\mathrm{D}\end{array}$} \\
\hline & & & & $\mathrm{A} \times 10^{7}$ & $\mathrm{~B} \times 10^{4}$ & $\mathrm{C} \times 10$ & D \\
\hline $2-\mathrm{Br}_{1}-1,3,6,7,8-\mathrm{Cl}_{5}-\mathrm{DD}$ & -105.0 & 27.4 & 596.6 & 1.716 & -6.489 & 8.722 & 73.43 \\
\hline $2-\mathrm{Br}_{1}-1,3,6,8,9-\mathrm{Cl}_{5}-\mathrm{DD}$ & -106.9 & 25.0 & 598.2 & 1.710 & -6.468 & 8.703 & 73.87 \\
\hline $2-\mathrm{Br}_{1}-1,3,7,8,9-\mathrm{Cl}_{5}-\mathrm{DD}$ & -103.7 & 28.7 & 596.6 & 1.717 & -6.492 & 8.725 & 73.37 \\
\hline 2- $\mathrm{Br}_{1}-1,4,7,8,9-\mathrm{Cl}_{5}-\mathrm{DD}$ & -105.6 & 26.5 & 597.9 & 1.708 & -6.465 & 8.702 & 73.73 \\
\hline $2-\mathrm{Br}_{1}-3,4,6,7,8-\mathrm{Cl}_{5}-\mathrm{DD}$ & -102.3 & 30.3 & 596.1 & 1.716 & -6.487 & 8.721 & 73.40 \\
\hline 2- $\mathrm{Br}_{1}-3,4,7,8,9-\mathrm{Cl}_{5}-\mathrm{DD}$ & -103.6 & 28.7 & 597.2 & 1.715 & -6.485 & 8.719 & 73.46 \\
\hline $2-\mathrm{Br}_{1}-3,6,7,8,9-\mathrm{Cl}_{5}-\mathrm{DD}$ & -101.8 & 30.6 & 596.5 & 1.714 & -6.483 & 8.719 & 73.37 \\
\hline $2-\mathrm{Br}_{1}-4,6,7,8,9-\mathrm{Cl}_{5}-\mathrm{DD}$ & -101.0 & 30.8 & 598.7 & 1.707 & -6.462 & 8.698 & 73.91 \\
\hline $1-\mathrm{Br}_{1}-2,3,4,6,7,8-\mathrm{Cl}_{6}-\mathrm{DD}$ & -107.2 & 30.5 & 625.5 & 1.725 & -6.482 & 8.600 & 92.45 \\
\hline $1-\mathrm{Br}_{1}-2,3,4,7,8,9-\mathrm{Cl}_{6}-\mathrm{DD}$ & -107.5 & 30.4 & 624.8 & 1.725 & -6.481 & 8.600 & 92.40 \\
\hline $1-\mathrm{Br}_{1}-2,3,6,7,8,9-\mathrm{Cl}_{6}-\mathrm{DD}$ & -107.4 & 30.5 & 624.8 & 1.723 & -6.477 & 8.596 & 92.57 \\
\hline $1-\mathrm{Br}_{1}-3,4,6,7,8,9-\mathrm{Cl}_{6}-\mathrm{DD}$ & -107.9 & 29.4 & 627.0 & 1.716 & -6.455 & 8.578 & 92.75 \\
\hline $2-\mathrm{Br}_{1}-1,3,4,6,7,8-\mathrm{Cl}_{6}-\mathrm{DD}$ & -107.0 & 31.3 & 623.6 & 1.724 & -6.477 & 8.595 & 92.68 \\
\hline $2-\mathrm{Br}_{1}-1,3,4,7,8,9-\mathrm{Cl}_{6}-\mathrm{DD}$ & -106.8 & 31.2 & 624.4 & 1.723 & -6.475 & 8.592 & 92.83 \\
\hline $2-\mathrm{Br}_{1}-1,3,6,7,8,9-\mathrm{Cl}_{6}-\mathrm{DD}$ & -106.8 & 31.0 & 625.3 & 1.723 & -6.474 & 8.591 & 92.84 \\
\hline $2-\mathrm{Br}_{1}-3,4,6,7,8,9-\mathrm{Cl}_{6}-\mathrm{DD}$ & -105.3 & 32.2 & 626.1 & 1.721 & -6.469 & 8.587 & 92.89 \\
\hline $1-\mathrm{Br}_{1}-2,3,4,6,7,8,9-\mathrm{Cl}_{7}-\mathrm{DD}$ & -109.2 & 33.8 & 654.3 & 1.730 & -6.464 & 8.467 & 111.9 \\
\hline $2-\mathrm{Br}_{1}-1,3,4,6,7,8,9-\mathrm{Cl}_{7}-\mathrm{DD}$ & -108.5 & 34.4 & 654.5 & 1.729 & -6.458 & 8.459 & 112.3 \\
\hline $1,2-\mathrm{Br}_{2}-3-\mathrm{Cl}_{1}-\mathrm{DD}$ & -6.4 & 96.8 & 519.1 & 1.678 & -6.482 & 9.080 & 15.54 \\
\hline $1,3-\mathrm{Br}_{2}-9-\mathrm{Cl}_{1}-\mathrm{DD}$ & -16.0 & 86.2 & 522.7 & 1.670 & -6.454 & 9.048 & 16.58 \\
\hline 1,4- $\mathrm{Br}_{2}-7-\mathrm{Cl}_{1}-\mathrm{DD}$ & -19.7 & 82.3 & 523.2 & 1.673 & -6.466 & 9.064 & 15.84 \\
\hline 1,6- $\mathrm{Br}_{2}-3-\mathrm{Cl}_{1}-\mathrm{DD}$ & -18.7 & 83.2 & 523.6 & 1.671 & -6.460 & 9.057 & 16.10 \\
\hline 1,7- $\mathrm{Br}_{2}-8-\mathrm{Cl}_{1}-\mathrm{DD}$ & -16.2 & 86.1 & 521.8 & 1.675 & -6.471 & 9.065 & 16.08 \\
\hline $1,8-\mathrm{Br}_{2}-6-\mathrm{Cl}_{1}-\mathrm{DD}$ & -17.0 & 85.0 & 523.2 & 1.669 & -6.451 & 9.045 & 16.64 \\
\hline $1,9-\mathrm{Br}_{2}-3-\mathrm{Cl}_{1}-\mathrm{DD}$ & -17.7 & 84.2 & 523.4 & 1.672 & -6.463 & 9.059 & 16.03 \\
\hline 2,3- $-\mathrm{Br}_{2}-7-\mathrm{Cl}_{1}-\mathrm{DD}$ & -24.8 & 77.8 & 520.9 & 1.677 & -6.474 & 9.060 & 16.79 \\
\hline 2,7- $\mathrm{Br}_{2}-4-\mathrm{Cl}_{1}-\mathrm{DD}$ & -22.3 & 79.8 & 522.8 & 1.671 & -6.454 & 9.041 & 17.29 \\
\hline $2,8-\mathrm{Br}_{2}-3-\mathrm{Cl}_{1}-\mathrm{DD}$ & -22.0 & 80.6 & 521.2 & 1.676 & -6.470 & 9.056 & 16.89 \\
\hline $1,2-\mathrm{Br}_{2}-3,4-\mathrm{Cl}_{2}-\mathrm{DD}$ & -11.1 & 97.7 & 547.2 & 1.685 & -6.469 & 8.951 & 34.84 \\
\hline $1,3-\mathrm{Br}_{2}-7,9-\mathrm{Cl}_{2}-\mathrm{DD}$ & -40.6 & 66.3 & 553.4 & 1.678 & -6.437 & 8.901 & 37.28 \\
\hline $1,4-\mathrm{Br}_{2}-6,9-\mathrm{Cl}_{2}-\mathrm{DD}$ & -28.2 & 79.1 & 552.2 & 1.673 & -6.430 & 8.911 & 35.81 \\
\hline $1,6-\mathrm{Br}_{2}-2,8-\mathrm{Cl}_{2}-\mathrm{DD}$ & -36.0 & 71.6 & 550.8 & 1.683 & -6.458 & 8.929 & 36.02 \\
\hline $1,7-\mathrm{Br}_{2}-3,9-\mathrm{Cl}_{2}-\mathrm{DD}$ & -40.5 & 66.3 & 553.3 & 1.678 & -6.438 & 8.902 & 37.22 \\
\hline $1,8-\mathrm{Br}_{2}-3,7-\mathrm{Cl}_{2}-\mathrm{DD}$ & -41.2 & 66.1 & 551.9 & 1.684 & -6.455 & 8.919 & 36.84 \\
\hline $1,9-\mathrm{Br}_{2}-2,7-\mathrm{Cl}_{2}-\mathrm{DD}$ & -35.1 & 72.4 & 551.2 & 1.684 & -6.460 & 8.930 & 36.03 \\
\hline $2,3-\mathrm{Br}_{2}-1,8-\mathrm{Cl}_{2}-\mathrm{DD}$ & -32.0 & 75.8 & 550.1 & 1.687 & -6.466 & 8.933 & 36.36 \\
\hline $2,3-\mathrm{Br}_{2}-7,8-\mathrm{Cl}_{2}-\mathrm{DD}$ & -40.2 & 67.9 & 549.4 & 1.686 & -6.463 & 8.927 & 36.62 \\
\hline $2,7-\mathrm{Br}_{2}-3,4-\mathrm{Cl}_{2}-\mathrm{DD}$ & -27.6 & 80.3 & 550.0 & 1.683 & -6.456 & 8.924 & 36.47 \\
\hline $2,7-\mathrm{Br}_{2}-3,8-\mathrm{Cl}_{2}-\mathrm{DD}$ & -38.9 & 69.0 & 549.9 & 1.686 & -6.464 & 8.926 & 36.24 \\
\hline $2,8-\mathrm{Br}_{2}-1,7-\mathrm{Cl}_{2}-\mathrm{DD}$ & -31.9 & 76.0 & 549.9 & 1.684 & -6.460 & 8.929 & 36.24 \\
\hline $2,8-\mathrm{Br}_{2}-3,7-\mathrm{Cl}_{2}-\mathrm{DD}$ & -39.1 & 69.0 & 549.0 & 1.687 & -6.467 & 8.930 & 36.53 \\
\hline $1,2-\mathrm{Br}_{2}-3,4,7-\mathrm{Cl}_{3}-\mathrm{DD}$ & -37.8 & 75.8 & 577.2 & 1.696 & -6.463 & 8.813 & 55.38 \\
\hline $1,2-\mathrm{Br}_{2}-3,7,8-\mathrm{Cl}_{3}-\mathrm{DD}$ & -48.6 & 64.8 & 578.4 & 1.698 & -6.464 & 8.808 & 55.95 \\
\hline $1,2-\mathrm{Br}_{2}-6,7,9-\mathrm{Cl}_{3}-\mathrm{DD}$ & -42.2 & 70.4 & 580.6 & 1.686 & -6.429 & 8.783 & 55.93 \\
\hline $1,3-\mathrm{Br}_{2}-2,4,6-\mathrm{Cl}_{3}-\mathrm{DD}$ & -28.1 & 85.1 & 578.6 & 1.692 & -6.452 & 8.809 & 55.08 \\
\hline $1,3-\mathrm{Br}_{2}-2,7,8-\mathrm{Cl}_{3}-\mathrm{DD}$ & -46.3 & 67.4 & 577.1 & 1.697 & -6.462 & 8.807 & 55.86 \\
\hline $1,3-\mathrm{Br}_{2}-7,8,9-\mathrm{Cl}_{3}-\mathrm{DD}$ & -43.8 & 68.9 & 580.4 & 1.689 & -6.436 & 8.783 & 56.38 \\
\hline $1,4-\mathrm{Br}_{2}-2,6,9-\mathrm{Cl}_{3}-\mathrm{DD}$ & -43.1 & 69.0 & 582.6 & 1.684 & -6.425 & 8.782 & 55.64 \\
\hline $1,4-\mathrm{Br}_{2}-6,7,8-\mathrm{Cl}_{3}-\mathrm{DD}$ & -38.9 & 73.8 & 580.7 & 1.687 & -6.437 & 8.793 & 55.40 \\
\hline $1,6-\mathrm{Br}_{2}-3,4,8-\mathrm{Cl}_{3}-\mathrm{DD}$ & -49.2 & 63.0 & 582.3 & 1.686 & -6.428 & 8.779 & 56.20 \\
\hline $1,7-\mathrm{Br}_{2}-2,3,8-\mathrm{Cl}_{3}-\mathrm{DD}$ & -46.0 & 67.6 & 577.6 & 1.697 & -6.462 & 8.808 & 55.80 \\
\hline $1,7-\mathrm{Br}_{2}-2,4,9-\mathrm{Cl}_{3}-\mathrm{DD}$ & -48.4 & 64.0 & 581.8 & 1.685 & -6.423 & 8.771 & 56.72 \\
\hline $1,7-\mathrm{Br}_{2}-3,4,8-\mathrm{Cl}_{3}-\mathrm{DD}$ & -47.7 & 64.8 & 581.0 & 1.689 & -6.439 & 8.788 & 56.10 \\
\hline $1,8-\mathrm{Br}_{2}-2,3,7-\mathrm{Cl}_{3}-\mathrm{DD}$ & -46.0 & 67.2 & 578.7 & 1.697 & -6.461 & 8.805 & 55.96 \\
\hline $1,8-\mathrm{Br}_{2}-3,4,6-\mathrm{Cl}_{3}-\mathrm{DD}$ & -47.0 & 65.0 & 582.6 & 1.683 & -6.419 & 8.769 & 56.63 \\
\hline
\end{tabular}




\begin{tabular}{|c|c|c|c|c|c|c|c|}
\hline \multirow{3}{*}{ Compound } & \multirow{3}{*}{$\begin{array}{c}\Delta_{\mathrm{f}} H^{\circ}(298 \mathrm{~K}) \\
\mathrm{kJ} \cdot \mathrm{mol}^{-1}\end{array}$} & \multirow{3}{*}{$\begin{array}{c}\Delta_{\mathrm{f}} G^{\circ}(298 \mathrm{~K}) \\
\mathrm{kJ} \cdot \mathrm{mol}^{-1}\end{array}$} & \multirow{3}{*}{$\begin{array}{c}S^{\circ}(298 \mathrm{~K}) \\
\mathrm{J} \cdot \mathrm{mol}^{-1} \cdot \mathrm{K}^{-1}\end{array}$} & \multirow{2}{*}{\multicolumn{4}{|c|}{$\begin{array}{c}C_{\mathrm{p}}, \mathrm{J} \cdot \mathrm{mol}^{-1} \cdot \mathrm{K}^{-1} \\
C_{\mathrm{p}}=\mathrm{A} T^{3}+\mathrm{B} T^{2}+\mathrm{C} T+\mathrm{D}\end{array}$}} \\
\hline & & & & & & & \\
\hline & & & & $\mathrm{A} \times 10^{7}$ & $\mathrm{~B} \times 10^{4}$ & $\mathrm{C} \times 10$ & $\mathrm{D}$ \\
\hline $1,8-\mathrm{Br}_{2}-6,7,9-\mathrm{Cl}_{3}-\mathrm{DD}$ & -26.3 & 87.4 & 577.0 & 1.689 & -6.445 & 8.804 & 54.96 \\
\hline $1,9-\mathrm{Br}_{2}-2,4,7-\mathrm{Cl}_{3}-\mathrm{DD}$ & -50.7 & 61.5 & 581.9 & 1.687 & -6.432 & 8.782 & 56.20 \\
\hline $2,3-\mathrm{Br}_{2}-1,7,8-\mathrm{Cl}_{3}-\mathrm{DD}$ & -46.7 & 66.6 & 578.4 & 1.696 & -6.456 & 8.799 & 56.26 \\
\hline $2,3-\mathrm{Br}_{2}-6,7,8-\mathrm{Cl}_{3}-\mathrm{DD}$ & -45.0 & 68.8 & 576.8 & 1.694 & -6.452 & 8.799 & 55.97 \\
\hline $2,7-\mathrm{Br}_{2}-1,3,8-\mathrm{Cl}_{3}-\mathrm{DD}$ & -45.6 & 67.7 & 578.3 & 1.696 & -6.457 & 8.800 & 56.21 \\
\hline $2,7-\mathrm{Br}_{2}-3,4,8-\mathrm{Cl}_{3}-\mathrm{DD}$ & -44.1 & 69.3 & 578.0 & 1.695 & -6.454 & 8.799 & 56.14 \\
\hline $2,8-\mathrm{Br}_{2}-1,3,7-\mathrm{Cl}_{3}-\mathrm{DD}$ & -45.6 & 67.8 & 578.3 & 1.696 & -6.457 & 8.800 & 56.25 \\
\hline $2,8-\mathrm{Br}_{2}-3,4,7-\mathrm{Cl}_{3}-\mathrm{DD}$ & -44.2 & 69.1 & 578.6 & 1.694 & -6.452 & 8.796 & 56.23 \\
\hline $1,2-\mathrm{Br}_{2}-3,4,7,8-\mathrm{Cl}_{4}-\mathrm{DD}$ & -52.1 & 66.5 & 607.0 & 1.706 & -6.453 & 8.680 & 75.30 \\
\hline $1,2-\mathrm{Br}_{2}-3,6,7,8-\mathrm{Cl}_{4}-\mathrm{DD}$ & -53.4 & 65.5 & 606.2 & 1.704 & -6.448 & 8.675 & 75.47 \\
\hline $1,2-\mathrm{Br}_{2}-3,7,8,9-\mathrm{Cl}_{4}-\mathrm{DD}$ & -52.4 & 66.4 & 606.6 & 1.705 & -6.450 & 8.677 & 75.38 \\
\hline $1,3-\mathrm{Br}_{2}-2,4,7,8-\mathrm{Cl}_{4}-\mathrm{DD}$ & -51.4 & 67.3 & 606.9 & 1.706 & -6.453 & 8.680 & 75.32 \\
\hline $1,3-\mathrm{Br}_{2}-2,6,7,8-\mathrm{Cl}_{4}-\mathrm{DD}$ & -51.2 & 67.9 & 605.7 & 1.704 & -6.446 & 8.673 & 75.40 \\
\hline $1,3-\mathrm{Br}_{2}-2,7,8,9-\mathrm{Cl}_{4}-\mathrm{DD}$ & -50.0 & 68.6 & 607.1 & 1.704 & -6.446 & 8.673 & 75.49 \\
\hline $1,3-\mathrm{Br}_{2}-4,7,8,9-\mathrm{Cl}_{4}-\mathrm{DD}$ & -52.1 & 66.2 & 608.3 & 1.696 & -6.424 & 8.657 & 75.49 \\
\hline $1,4-\mathrm{Br}_{2}-2,3,7,8-\mathrm{Cl}_{4}-\mathrm{DD}$ & -51.5 & 67.3 & 606.6 & 1.707 & -6.460 & 8.690 & 74.78 \\
\hline $1,4-\mathrm{Br}_{2}-6,7,8,9-\mathrm{Cl}_{4}-\mathrm{DD}$ & -41.2 & 76.7 & 609.9 & 1.693 & -6.420 & 8.661 & 74.78 \\
\hline $1,6-\mathrm{Br}_{2}-2,3,7,8-\mathrm{Cl}_{4}-\mathrm{DD}$ & -51.5 & 67.3 & 606.6 & 1.707 & -6.456 & 8.684 & 75.06 \\
\hline $1,6-\mathrm{Br}_{2}-2,4,7,9-\mathrm{Cl}_{4}-\mathrm{DD}$ & -57.2 & 60.6 & 610.0 & 1.694 & -6.419 & 8.651 & 75.65 \\
\hline $1,7-\mathrm{Br}_{2}-2,3,4,8-\mathrm{Cl}_{4}-\mathrm{DD}$ & -49.4 & 69.4 & 606.8 & 1.704 & -6.446 & 8.675 & 75.32 \\
\hline $1,7-\mathrm{Br}_{2}-2,3,6,8-\mathrm{Cl}_{4}-\mathrm{DD}$ & -52.4 & 66.6 & 606.0 & 1.706 & -6.452 & 8.679 & 75.33 \\
\hline $1,7-\mathrm{Br}_{2}-2,3,8,9-\mathrm{Cl}_{4}-\mathrm{DD}$ & -50.0 & 68.6 & 607.3 & 1.704 & -6.448 & 8.675 & 75.39 \\
\hline $1,7-\mathrm{Br}_{2}-3,4,8,9-\mathrm{Cl}_{4}-\mathrm{DD}$ & -51.8 & 66.2 & 609.2 & 1.697 & -6.425 & 8.657 & 75.60 \\
\hline $1,8-\mathrm{Br}_{2}-2,3,4,7-\mathrm{Cl}_{4}-\mathrm{DD}$ & -49.2 & 69.6 & 606.5 & 1.704 & -6.448 & 8.677 & 75.20 \\
\hline $1,8-\mathrm{Br}_{2}-2,3,6,7-\mathrm{Cl}_{4}-\mathrm{DD}$ & -50.9 & 67.9 & 606.7 & 1.704 & -6.446 & 8.674 & 75.37 \\
\hline $1,8-\mathrm{Br}_{2}-2,3,7,9-\mathrm{Cl}_{4}-\mathrm{DD}$ & -51.4 & 67.3 & 607.0 & 1.706 & -6.453 & 8.678 & 75.43 \\
\hline $1,8-\mathrm{Br}_{2}-2,4,7,9-\mathrm{Cl}_{4}-\mathrm{DD}$ & -54.6 & 64.0 & 607.6 & 1.699 & -6.432 & 8.661 & 75.67 \\
\hline $1,9-\mathrm{Br}_{2}-2,3,7,8-\mathrm{Cl}_{4}-\mathrm{DD}$ & -52.2 & 66.6 & 606.5 & 1.706 & -6.455 & 8.682 & 75.15 \\
\hline $1,9-\mathrm{Br}_{2}-2,4,6,8-\mathrm{Cl}_{4}-\mathrm{DD}$ & -57.6 & 60.2 & 610.1 & 1.693 & -6.415 & 8.647 & 75.81 \\
\hline $2,3-\mathrm{Br}_{2}-1,4,7,8-\mathrm{Cl}_{4}-\mathrm{DD}$ & -51.9 & 67.3 & 605.4 & 1.704 & -6.448 & 8.674 & 75.57 \\
\hline $2,3-\mathrm{Br}_{2}-1,6,7,8-\mathrm{Cl}_{4}-\mathrm{DD}$ & -51.6 & 67.3 & 606.4 & 1.702 & -6.441 & 8.667 & 75.69 \\
\hline $2,3-\mathrm{Br}_{2}-1,7,8,9-\mathrm{Cl}_{4}-\mathrm{DD}$ & -50.4 & 68.6 & 606.1 & 1.703 & -6.444 & 8.670 & 75.62 \\
\hline $2,3-\mathrm{Br}_{2}-6,7,8,9-\mathrm{Cl}_{4}-\mathrm{DD}$ & -48.2 & 70.9 & 605.5 & 1.699 & -6.434 & 8.665 & 75.46 \\
\hline $2,7-\mathrm{Br}_{2}-1,3,4,8-\mathrm{Cl}_{4}-\mathrm{DD}$ & -48.9 & 69.8 & 606.8 & 1.702 & -6.441 & 8.668 & 75.65 \\
\hline $2,7-\mathrm{Br}_{2}-1,3,6,8-\mathrm{Cl}_{4}-\mathrm{DD}$ & -52.1 & 67.0 & 605.9 & 1.705 & -6.448 & 8.673 & 75.62 \\
\hline $2,7-\mathrm{Br}_{2}-1,3,8,9-\mathrm{Cl}_{4}-\mathrm{DD}$ & -49.5 & 69.6 & 605.8 & 1.704 & -6.447 & 8.673 & 75.51 \\
\hline $2,7-\mathrm{Br}_{2}-3,4,8,9-\mathrm{Cl}_{4}-\mathrm{DD}$ & -49.0 & 69.6 & 607.1 & 1.701 & -6.437 & 8.665 & 75.71 \\
\hline $2,8-\mathrm{Br}_{2}-1,3,4,7-\mathrm{Cl}_{4}-\mathrm{DD}$ & -49.0 & 69.9 & 606.5 & 1.703 & -6.443 & 8.670 & 75.59 \\
\hline $2,8-\mathrm{Br}_{2}-1,3,6,7-\mathrm{Cl}_{4}-\mathrm{DD}$ & -50.7 & 68.3 & 606.0 & 1.703 & -6.444 & 8.670 & 75.56 \\
\hline $2,8-\mathrm{Br}_{2}-1,3,7,9-\mathrm{Cl}_{4}-\mathrm{DD}$ & -50.9 & 68.2 & 605.6 & 1.705 & -6.450 & 8.675 & 75.55 \\
\hline $2,8-\mathrm{Br}_{2}-3,4,6,7-\mathrm{Cl}_{4}-\mathrm{DD}$ & -48.0 & 71.0 & 605.9 & 1.702 & -6.442 & 8.670 & 75.48 \\
\hline $1,2-\mathrm{Br}_{2}-3,4,6,7,8-\mathrm{Cl}_{5}-\mathrm{DD}$ & -55.5 & 68.8 & 634.7 & 1.713 & -6.439 & 8.549 & 94.74 \\
\hline $1,2-\mathrm{Br}_{2}-3,6,7,8,9-\mathrm{Cl}_{5}-\mathrm{DD}$ & -55.3 & 68.8 & 635.6 & 1.711 & -6.433 & 8.543 & 94.92 \\
\hline $1,3-\mathrm{Br}_{2}-2,4,7,8,9-\mathrm{Cl}_{5}-\mathrm{DD}$ & -54.7 & 69.2 & 636.0 & 1.712 & -6.438 & 8.547 & 94.85 \\
\hline $1,3-\mathrm{Br}_{2}-2,6,7,8,9-\mathrm{Cl}_{5}-\mathrm{DD}$ & -53.0 & 71.0 & 635.8 & 1.709 & -6.428 & 8.539 & 94.99 \\
\hline $1,4-\mathrm{Br}_{2}-2,3,6,7,8-\mathrm{Cl}_{5}-\mathrm{DD}$ & -55.1 & 68.8 & 636.2 & 1.714 & -6.445 & 8.557 & 94.33 \\
\hline $1,6-\mathrm{Br}_{2}-2,3,4,7,8-\mathrm{Cl}_{5}-\mathrm{DD}$ & -55.0 & 69.0 & 635.6 & 1.714 & -6.443 & 8.554 & 94.51 \\
\hline $1,7-\mathrm{Br}_{2}-2,3,4,6,8-\mathrm{Cl}_{5}-\mathrm{DD}$ & -54.3 & 69.9 & 634.8 & 1.713 & -6.439 & 8.549 & 94.74 \\
\hline $1,7-\mathrm{Br}_{2}-2,3,6,8,9-\mathrm{Cl}_{5}-\mathrm{DD}$ & -54.5 & 69.6 & 635.3 & 1.712 & -6.436 & 8.546 & 94.86 \\
\hline $1,8-\mathrm{Br}_{2}-2,3,4,7,9-\mathrm{Cl}_{5}-\mathrm{DD}$ & -54.6 & 69.4 & 635.8 & 1.712 & -6.438 & 8.548 & 94.76 \\
\hline $1,8-\mathrm{Br}_{2}-2,3,6,7,9-\mathrm{Cl}_{5}-\mathrm{DD}$ & -54.5 & 69.4 & 636.0 & 1.712 & -6.436 & 8.545 & 94.93 \\
\hline $1,9-\mathrm{Br}_{2}-2,3,4,7,8-\mathrm{Cl}_{5}-\mathrm{DD}$ & -55.3 & 68.6 & 635.9 & 1.713 & -6.441 & 8.553 & 94.48 \\
\hline $2,3-\mathrm{Br}_{2}-1,6,7,8,9-\mathrm{Cl}_{5}-\mathrm{DD}$ & -53.3 & 71.3 & 633.7 & 1.709 & -6.427 & 8.538 & 94.96 \\
\hline $2,7-\mathrm{Br}_{2}-1,3,4,6,8-\mathrm{Cl}_{5}-\mathrm{DD}$ & -53.9 & 70.6 & 634.3 & 1.711 & -6.433 & 8.543 & 95.01 \\
\hline $2,8-\mathrm{Br}_{2}-1,3,4,7,9-\mathrm{Cl}_{5}-\mathrm{DD}$ & -53.8 & 70.3 & 635.3 & 1.711 & -6.433 & 8.541 & 95.16 \\
\hline
\end{tabular}




\begin{tabular}{|c|c|c|c|c|c|c|c|}
\hline \multirow{2}{*}{ Compound } & \multirow{2}{*}{$\begin{array}{c}\Delta_{\mathrm{f}} H^{\circ}(298 \mathrm{~K}) \\
\mathrm{kJ} \cdot \mathrm{mol}^{-1}\end{array}$} & \multirow{2}{*}{$\begin{array}{c}\Delta_{\mathrm{f}} G^{\circ}(298 \mathrm{~K}) \\
\mathrm{kJ} \cdot \mathrm{mol}^{-1}\end{array}$} & \multirow{2}{*}{$\begin{array}{c}S^{\circ}(298 \mathrm{~K}) \\
\mathrm{J} \cdot \mathrm{mol}^{-1} \cdot \mathrm{K}^{-1}\end{array}$} & \multicolumn{4}{|c|}{$\begin{array}{c}C_{\mathrm{p}}, \mathrm{J} \cdot \mathrm{mol}^{-1} \cdot \mathrm{K}^{-1} \\
C_{\mathrm{p}}=\mathrm{A} T^{3}+\mathrm{B} T^{2}+\mathrm{C} T+\mathrm{D}\end{array}$} \\
\hline & & & & $\mathrm{A} \times 10^{7}$ & $\mathrm{~B} \times 10^{4}$ & & $\mathrm{D}$ \\
\hline $1,4-\mathrm{Br}_{2}-2,3,6,7,8,9-\mathrm{Cl}_{6}-\mathrm{DD}$ & -57.0 & 72.1 & 665.4 & 1.720 & -6.427 & 8.422 & 113.9 \\
\hline $1,7-\mathrm{Br}_{2}-2,3,4,6,8,9-\mathrm{Cl}_{6}-\mathrm{DD}$ & -56.5 & 73.1 & 663.9 & 1.719 & -6.424 & 8.418 & 114.1 \\
\hline $2,3-\mathrm{Br}_{2}-1,4,6,7,8,9-\mathrm{Cl}_{6}-\mathrm{DD}$ & -56.7 & 72.8 & 664.0 & 1.716 & -6.414 & 8.406 & 114.7 \\
\hline $2,8-\mathrm{Br}_{2}-1,3,4,6,7,9-\mathrm{Cl}_{6}-\mathrm{DD}$ & -55.9 & 73.8 & 663.2 & 1.718 & -6.419 & 8.411 & 114.5 \\
\hline $1,2,3-\mathrm{Br}_{3}-4-\mathrm{Cl}_{1}-\mathrm{DD}$ & 40.9 & 135.8 & 557.9 & 1.672 & -6.424 & 8.897 & 37.28 \\
\hline $1,2,6-\mathrm{Br}_{3}-7-\mathrm{Cl}_{1}-\mathrm{DD}$ & 25.2 & 119.2 & 560.8 & 1.672 & -6.421 & 8.890 & 37.56 \\
\hline $1,3,7-\mathrm{Br}_{3}-2-\mathrm{Cl}_{1}-\mathrm{DD}$ & 25.1 & 118.9 & 561.4 & 1.671 & -6.414 & 8.874 & 38.62 \\
\hline $1,4,6-\mathrm{Br}_{3}-9-\mathrm{Cl}_{1}-\mathrm{DD}$ & 26.0 & 118.7 & 565.4 & 1.659 & -6.385 & 8.862 & 37.75 \\
\hline $2,3,7-\mathrm{Br}_{3}-8-\mathrm{Cl}_{1}-\mathrm{DD}$ & 14.5 & 108.5 & 561.0 & 1.672 & -6.415 & 8.871 & 39.03 \\
\hline $1,2,3-\mathrm{Br}_{3}-7,8-\mathrm{Cl}_{2}-\mathrm{DD}$ & 4.7 & 104.6 & 588.0 & 1.683 & -6.413 & 8.750 & 58.23 \\
\hline $1,2,4-\mathrm{Br}_{3}-6,9-\mathrm{Cl}_{2}-\mathrm{DD}$ & 10.0 & 108.7 & 591.8 & 1.669 & -6.374 & 8.725 & 57.96 \\
\hline $1,3,7-\mathrm{Br}_{3}-2,4-\mathrm{Cl}_{2}-\mathrm{DD}$ & 19.6 & 119.2 & 588.6 & 1.680 & -6.406 & 8.750 & 57.88 \\
\hline $1,3,7-\mathrm{Br}_{3}-2,8-\mathrm{Cl}_{2}-\mathrm{DD}$ & 8.3 & 107.9 & 588.8 & 1.682 & -6.410 & 8.747 & 58.37 \\
\hline $2,3,7-\mathrm{Br}_{3}-8,9-\mathrm{Cl}_{2}-\mathrm{DD}$ & 9.4 & 108.7 & 589.7 & 1.679 & -6.401 & 8.739 & 58.53 \\
\hline $2,3,8-\mathrm{Br}_{3}-1,7-\mathrm{Cl}_{2}-\mathrm{DD}$ & 7.8 & 107.2 & 589.1 & 1.681 & -6.407 & 8.743 & 58.57 \\
\hline $1,2,3-\mathrm{Br}_{3}-6,7,8-\mathrm{Cl}_{3}-\mathrm{DD}$ & -0.1 & 105.1 & 616.5 & 1.690 & -6.398 & 8.618 & 77.80 \\
\hline $1,2,4-\mathrm{Br}_{3}-6,7,9-\mathrm{Cl}_{3}-\mathrm{DD}$ & -2.6 & 100.6 & 623.4 & 1.676 & -6.360 & 8.588 & 77.97 \\
\hline $1,2,6-\mathrm{Br}_{3}-4,8,9-\mathrm{Cl}_{3}-\mathrm{DD}$ & -2.6 & 101.0 & 622.1 & 1.678 & -6.364 & 8.592 & 77.87 \\
\hline $1,3,7-\mathrm{Br}_{3}-2,4,8-\mathrm{Cl}_{3}-\mathrm{DD}$ & 3.3 & 108.8 & 615.7 & 1.692 & -6.405 & 8.627 & 77.45 \\
\hline $1,3,7-\mathrm{Br}_{3}-2,6,8-\mathrm{Cl}_{3}-\mathrm{DD}$ & 2.0 & 106.8 & 617.9 & 1.693 & -6.409 & 8.628 & 77.52 \\
\hline $2,3,7-\mathrm{Br}_{3}-1,6,8-\mathrm{Cl}_{3}-\mathrm{DD}$ & 1.3 & 105.9 & 618.7 & 1.689 & -6.396 & 8.613 & 78.11 \\
\hline $1,2,3-\mathrm{Br}_{3}-4,6,7,8-\mathrm{Cl}_{4}-\mathrm{DD}$ & -3.6 & 106.9 & 645.3 & 1.699 & -6.392 & 8.493 & 97.20 \\
\hline $1,2,4-\mathrm{Br}_{3}-3,7,8,9-\mathrm{Cl}_{4}-\mathrm{DD}$ & -3.3 & 107.3 & 644.9 & 1.701 & -6.399 & 8.502 & 96.74 \\
\hline $1,2,7-\mathrm{Br}_{3}-3,4,6,8-\mathrm{Cl}_{4}-\mathrm{DD}$ & -2.6 & 107.8 & 645.8 & 1.700 & -6.396 & 8.498 & 97.00 \\
\hline $1,3,7-\mathrm{Br}_{3}-2,4,6,8-\mathrm{Cl}_{4}-\mathrm{DD}$ & -1.5 & 109.1 & 644.8 & 1.700 & -6.396 & 8.498 & 97.03 \\
\hline $1,3,8-\mathrm{Br}_{3}-2,4,7,9-\mathrm{Cl}_{4}-\mathrm{DD}$ & -2.0 & 108.3 & 646.2 & 1.700 & -6.394 & 8.495 & 97.12 \\
\hline $2,3,7-\mathrm{Br}_{3}-1,4,6,8-\mathrm{Cl}_{4}-\mathrm{DD}$ & -2.1 & 108.5 & 645.1 & 1.698 & -6.389 & 8.488 & 97.49 \\
\hline $1,2,3-\mathrm{Br}_{3}-4,6,7,8,9-\mathrm{Cl}_{5}-\mathrm{DD}$ & -5.6 & 109.7 & 675.8 & 1.704 & -6.373 & 8.359 & 116.7 \\
\hline $1,2,4-\mathrm{Br}_{3}-3,6,7,8,9-\mathrm{Cl}_{5}-\mathrm{DD}$ & -5.3 & 110.6 & 673.7 & 1.706 & -6.381 & 8.369 & 116.2 \\
\hline $1,2,6-\mathrm{Br}_{3}-3,4,7,8,9-\mathrm{Cl}_{5}-\mathrm{DD}$ & -5.3 & 110.2 & 675.3 & 1.708 & -6.386 & 8.374 & 116.0 \\
\hline $1,2,7-\mathrm{Br}_{3}-3,4,6,8,9-\mathrm{Cl}_{5}-\mathrm{DD}$ & -4.7 & 111.0 & 674.4 & 1.706 & -6.380 & 8.365 & 116.5 \\
\hline $1,3,7-\mathrm{Br}_{3}-2,4,6,8,9-\mathrm{Cl}_{5}-\mathrm{DD}$ & -3.8 & 111.7 & 675.5 & 1.706 & -6.378 & 8.364 & 116.5 \\
\hline $2,3,7-\mathrm{Br}_{3}-1,4,6,8,9-\mathrm{Cl}_{5}-\mathrm{DD}$ & -4.1 & 111.0 & 676.5 & 1.704 & -6.372 & 8.354 & 117.0 \\
\hline $1,2,3,4-\mathrm{Br}_{4}-7-\mathrm{Cl}_{1}-\mathrm{DD}$ & 65.5 & 152.2 & 596.3 & 1.671 & -6.375 & 8.711 & 59.75 \\
\hline $1,2,3,7-\mathrm{Br}_{4}-8-\mathrm{Cl}_{1}-\mathrm{DD}$ & 59.4 & 145.6 & 598.2 & 1.669 & -6.366 & 8.696 & 60.52 \\
\hline $1,3,7,8-\mathrm{Br}_{4}-2-\mathrm{Cl}_{1}-\mathrm{DD}$ & 61.9 & 147.8 & 598.9 & 1.668 & -6.362 & 8.693 & 60.50 \\
\hline $1,4,6,9-\mathrm{Br}_{4}-2-\mathrm{Cl}_{1}-\mathrm{DD}$ & 65.0 & 148.8 & 605.9 & 1.657 & -6.338 & 8.689 & 59.21 \\
\hline $2,3,7,8-\mathrm{Br}_{4}-1-\mathrm{Cl}_{1}-\mathrm{DD}$ & 61.6 & 147.6 & 598.7 & 1.667 & -6.359 & 8.689 & 60.73 \\
\hline $1,2,3,4-\mathrm{Br}_{4}-7,8-\mathrm{Cl}_{2}-\mathrm{DD}$ & 51.1 & 142.7 & 626.5 & 1.680 & -6.364 & 8.576 & 79.74 \\
\hline $1,2,4,7-\mathrm{Br}_{4}-3,8-\mathrm{Cl}_{2}-\mathrm{DD}$ & 54.8 & 146.0 & 627.7 & 1.680 & -6.366 & 8.579 & 79.59 \\
\hline $1,2,6,7-\mathrm{Br}_{4}-3,8-\mathrm{Cl}_{2}-\mathrm{DD}$ & 51.2 & 143.0 & 625.8 & 1.681 & -6.367 & 8.579 & 79.59 \\
\hline $1,3,7,9-\mathrm{Br}_{4}-2,8-\mathrm{Cl}_{2}-\mathrm{DD}$ & 56.4 & 147.9 & 627.1 & 1.678 & -6.357 & 8.570 & 79.80 \\
\hline $1,4,6,9-\mathrm{Br}_{4}-2,7-\mathrm{Cl}_{2}-\mathrm{DD}$ & 50.0 & 139.8 & 632.3 & 1.668 & -6.334 & 8.559 & 79.13 \\
\hline $2,3,7,8-\mathrm{Br}_{4}-1,6-\mathrm{Cl}_{2}-\mathrm{DD}$ & 54.9 & 146.2 & 627.4 & 1.675 & -6.348 & 8.559 & 80.29 \\
\hline $1,2,3,4-\mathrm{Br}_{4}-6,7,8-\mathrm{Cl}_{3}-\mathrm{DD}$ & 47.6 & 144.3 & 656.0 & 1.686 & -6.349 & 8.443 & 99.31 \\
\hline $1,2,4,8-\mathrm{Br}_{4}-3,7,9-\mathrm{Cl}_{3}-\mathrm{DD}$ & 49.5 & 146.2 & 656.0 & 1.689 & -6.355 & 8.450 & 99.06 \\
\hline $1,2,6,7-\mathrm{Br}_{4}-3,4,8-\mathrm{Cl}_{3}-\mathrm{DD}$ & 48.8 & 145.9 & 654.7 & 1.689 & -6.356 & 8.451 & 98.96 \\
\hline $1,3,6,8-\mathrm{Br}_{4}-2,4,7-\mathrm{Cl}_{3}-\mathrm{DD}$ & 52.1 & 148.6 & 656.5 & 1.687 & -6.350 & 8.445 & 99.24 \\
\hline $1,4,6,9-\mathrm{Br}_{4}-2,3,7-\mathrm{Cl}_{3}-\mathrm{DD}$ & 48.3 & 143.6 & 660.6 & 1.683 & -6.343 & 8.448 & 98.28 \\
\hline $2,3,7,8-\mathrm{Br}_{4}-1,4,6-\mathrm{Cl}_{3}-\mathrm{DD}$ & 51.4 & 148.4 & 655.1 & 1.684 & -6.345 & 8.441 & 99.46 \\
\hline $1,2,3,4-\mathrm{Br}_{4}-6,7,8,9-\mathrm{Cl}_{4}-\mathrm{DD}$ & 45.4 & 147.4 & 685.1 & 1.692 & -6.331 & 8.310 & 118.8 \\
\hline $1,2,4,7-\mathrm{Br}_{4}-3,6,8,9-\mathrm{Cl}_{4}-\mathrm{DD}$ & 47.5 & 148.6 & 687.9 & 1.694 & -6.338 & 8.317 & 118.6 \\
\hline $1,2,6,7-\mathrm{Br}_{4}-3,4,8,9-\mathrm{Cl}_{4}-\mathrm{DD}$ & 46.3 & 148.6 & 684.1 & 1.696 & -6.345 & 8.324 & 118.2 \\
\hline $1,3,6,8-\mathrm{Br}_{4}-2,4,7,9-\mathrm{Cl}_{4}-\mathrm{DD}$ & 48.5 & 150.4 & 685.0 & 1.696 & -6.343 & 8.322 & 118.4 \\
\hline $1,4,6,9-\mathrm{Br}_{4}-2,3,7,8-\mathrm{Cl}_{4}-\mathrm{DD}$ & 46.7 & 148.2 & 686.3 & 1.698 & -6.353 & 8.336 & 117.6 \\
\hline
\end{tabular}




\begin{tabular}{|c|c|c|c|c|c|c|c|}
\hline \multirow{3}{*}{ Compound } & \multirow{3}{*}{$\begin{array}{c}\Delta_{\mathrm{f}} H^{\circ}(298 \mathrm{~K}) \\
\mathrm{kJ} \cdot \mathrm{mol}^{-1}\end{array}$} & \multirow{3}{*}{$\begin{array}{c}\Delta_{\mathrm{f}} G^{\circ}(298 \mathrm{~K}) \\
\mathrm{kJ} \cdot \mathrm{mol}^{-1}\end{array}$} & \multirow{3}{*}{$\begin{array}{c}S^{\circ}(298 \mathrm{~K}) \\
\mathrm{J} \cdot \mathrm{mol}^{-1} \cdot \mathrm{K}^{-1}\end{array}$} & \multirow{2}{*}{\multicolumn{4}{|c|}{$\begin{array}{c}C_{\mathrm{p}}, \mathrm{J} \cdot \mathrm{mol}^{-1} \cdot \mathrm{K}^{-1} \\
C_{\mathrm{p}}=\mathrm{A} T^{3}+\mathrm{B} T^{2}+\mathrm{C} T+\mathrm{D}\end{array}$}} \\
\hline & & & & & & & \\
\hline & & & & $\mathrm{A} \times 10^{7}$ & $\mathrm{~B} \times 10^{4}$ & $\mathrm{C} \times 10$ & D \\
\hline $2,3,7,8-\mathrm{Br}_{4}-1,4,6,9-\mathrm{Cl}_{4}-\mathrm{DD}$ & 47.9 & 150.0 & 684.6 & 1.692 & -6.328 & 8.302 & 119.4 \\
\hline $1,2,3,4,7-\mathrm{Br}_{5}-8-\mathrm{Cl}_{1}-\mathrm{DD}$ & 105.8 & 183.4 & 637.7 & 1.665 & -6.315 & 8.519 & 82.14 \\
\hline $1,2,3,6,7-\mathrm{Br}_{5}-8-\mathrm{Cl}_{1}-\mathrm{DD}$ & 104.6 & 181.9 & 638.9 & 1.665 & -6.312 & 8.517 & 82.15 \\
\hline $1,2,3,7,8-\mathrm{Br}_{5}-6-\mathrm{Cl}_{1}-\mathrm{DD}$ & 106.5 & 184.2 & 637.3 & 1.663 & -6.306 & 8.510 & 82.40 \\
\hline $1,2,4,6,9-\mathrm{Br}_{5}-7-\mathrm{Cl}_{1}-\mathrm{DD}$ & 103.2 & 178.2 & 646.3 & 1.653 & -6.282 & 8.502 & 81.42 \\
\hline $1,2,4,7,8-\mathrm{Br}_{5}-3-\mathrm{Cl}_{1}-\mathrm{DD}$ & 108.6 & 186.0 & 638.2 & 1.665 & -6.315 & 8.522 & 81.86 \\
\hline $1,2,3,4,6-\mathrm{Br}_{5}-7,8-\mathrm{Cl}_{2}-\mathrm{DD}$ & 99.6 & 182.5 & 666.9 & 1.675 & -6.309 & 8.396 & 101.3 \\
\hline $1,2,3,6,7-\mathrm{Br}_{5}-4,8-\mathrm{Cl}_{2}-\mathrm{DD}$ & 100.6 & 184.0 & 665.0 & 1.675 & -6.308 & 8.395 & 101.4 \\
\hline $1,2,3,7,8-\mathrm{Br}_{5}-6,9-\mathrm{Cl}_{2}-\mathrm{DD}$ & 102.4 & 185.8 & 664.8 & 1.671 & -6.297 & 8.383 & 101.8 \\
\hline $1,2,4,6,8-\mathrm{Br}_{5}-3,7-\mathrm{Cl}_{2}-\mathrm{DD}$ & 103.1 & 186.0 & 666.6 & 1.675 & -6.309 & 8.398 & 101.1 \\
\hline $1,2,4,6,9-\mathrm{Br}_{5}-3,7-\mathrm{Cl}_{2}-\mathrm{DD}$ & 100.1 & 181.9 & 670.4 & 1.669 & -6.295 & 8.390 & 101.0 \\
\hline $1,2,4,7,9-\mathrm{Br}_{5}-3,8-\mathrm{Cl}_{2}-\mathrm{DD}$ & 102.9 & 185.6 & 667.4 & 1.674 & -6.308 & 8.397 & 101.2 \\
\hline $1,2,3,4,6-\mathrm{Br}_{5}-7,8,9-\mathrm{Cl}_{3}-\mathrm{DD}$ & 97.3 & 185.6 & 695.1 & 1.682 & -6.296 & 8.268 & 120.6 \\
\hline $1,2,3,7,8-\mathrm{Br}_{5}-4,6,9-\mathrm{Cl}_{3}-\mathrm{DD}$ & 99.1 & 187.7 & 694.1 & 1.680 & -6.289 & 8.257 & 121.3 \\
\hline $1,2,3,8,9-\mathrm{Br}_{5}-4,6,7-\mathrm{Cl}_{3}-\mathrm{DD}$ & 97.9 & 185.9 & 696.2 & 1.681 & -6.294 & 8.264 & 120.9 \\
\hline $1,2,4,6,8-\mathrm{Br}_{5}-3,7,9-\mathrm{Cl}_{3}-\mathrm{DD}$ & 99.2 & 186.8 & 697.2 & 1.683 & -6.301 & 8.273 & 120.4 \\
\hline $1,2,4,6,9-\mathrm{Br}_{5}-3,7,8-\mathrm{Cl}_{3}-\mathrm{DD}$ & 98.3 & 185.7 & 698.2 & 1.685 & -6.307 & 8.283 & 119.9 \\
\hline $1,2,4,8,9-\mathrm{Br}_{5}-3,6,7-\mathrm{Cl}_{3}-\mathrm{DD}$ & 98.4 & 186.3 & 696.4 & 1.683 & -6.301 & 8.273 & 120.5 \\
\hline $1,2,3,4,6,7-\mathrm{Br}_{6}-8-\mathrm{Cl}_{1}-\mathrm{DD}$ & 151.5 & 220.7 & 676.7 & 1.661 & -6.263 & 8.343 & 103.6 \\
\hline $1,2,3,4,6,9-\mathrm{Br}_{6}-7-\mathrm{Cl}_{1}-\mathrm{DD}$ & 151.1 & 219.3 & 680.2 & 1.655 & -6.244 & 8.330 & 103.5 \\
\hline $1,2,3,4,7,8-\mathrm{Br}_{6}-6-\mathrm{Cl}_{1}-\mathrm{DD}$ & 153.8 & 222.5 & 678.7 & 1.659 & -6.256 & 8.335 & 103.9 \\
\hline $1,2,3,6,7,8-\mathrm{Br}_{6}-4-\mathrm{Cl}_{1}-\mathrm{DD}$ & 154.1 & 223.7 & 675.4 & 1.660 & -6.257 & 8.337 & 103.8 \\
\hline $1,2,4,6,8,9-\mathrm{Br}_{6}-3-\mathrm{Cl}_{1}-\mathrm{DD}$ & 153.2 & 221.1 & 681.1 & 1.654 & -6.243 & 8.332 & 103.2 \\
\hline $1,2,3,4,6,7-\mathrm{Br}_{6}-8,9-\mathrm{Cl}_{2}-\mathrm{DD}$ & 149.2 & 224.0 & 704.7 & 1.669 & -6.252 & 8.216 & 122.9 \\
\hline $1,2,3,4,6,9-\mathrm{Br}_{6}-7,8-\mathrm{Cl}_{2}-\mathrm{DD}$ & 149.2 & 223.2 & 707.3 & 1.670 & -6.257 & 8.223 & 122.5 \\
\hline $1,2,3,4,7,8-\mathrm{Br}_{6}-6,9-\mathrm{Cl}_{2}-\mathrm{DD}$ & 150.0 & 223.6 & 708.7 & 1.667 & -6.245 & 8.206 & 123.5 \\
\hline $1,2,3,6,7,8-\mathrm{Br}_{6}-4,9-\mathrm{Cl}_{2}-\mathrm{DD}$ & 150.1 & 224.8 & 705.0 & 1.668 & -6.249 & 8.210 & 123.3 \\
\hline $1,2,4,6,7,9-\mathrm{Br}_{6}-3,8-\mathrm{Cl}_{2}-\mathrm{DD}$ & 149.9 & 224.2 & 706.4 & 1.671 & -6.261 & 8.227 & 122.3 \\
\hline $1,2,3,4,6,7,8-\mathrm{Br}_{7}-9-\mathrm{Cl}_{1}-\mathrm{DD}$ & 201.1 & 262.1 & 715.4 & 1.655 & -6.204 & 8.159 & 125.4 \\
\hline $1,2,3,4,6,7,9-\mathrm{Br}_{7}-8-\mathrm{Cl}_{1}-\mathrm{DD}$ & 200.8 & 259.8 & 721.8 & 1.656 & -6.209 & 8.167 & 124.9 \\
\hline
\end{tabular}

${ }^{a}$ V. P. Kolesov, O. V. Dorofeeva, V. S. Iorish, T. S. Papina, V. A. Luckyanova and S. V. Melkhanova: Organohalogen Compounds 36 (1998), $201-204$. 